\title{
A subadult individual of Styracosaurus albertensis (Ornithischia: Ceratopsidae) with comments on ontogeny and intraspecific variation in Styracosaurus and Centrosaurus
}

\author{
Caleb M. Brown ${ }^{1, \star}$, Robert B. Holmes², Philip J. Currie ${ }^{2}$ \\ ${ }^{1}$ Royal Tyrrell Museum of Palaeontology, Box 7500, Drumheller, AB, T0J 0Y0, Canada; caleb.brown@gov.ab.ca \\ ${ }^{2}$ Department of Biological Sciences, University of Alberta, Edmonton, Alberta, T6G 2E9, Canada; \\ holmes1@ualberta.ca; philip.currie@ualberta.ca
}

\begin{abstract}
Styracosaurus albertensis is an iconic centrosaurine horned dinosaur from the Campanian of Alberta, Canada, known for its large spike-like parietal processes. Although described over 100 years ago, subsequent discoveries were rare until the last few decades, during which time several new skulls, skeletons, and bonebeds were found. Here we described an immature individual, the smallest known for the species, represented by a complete skull and fragmentary skeleton. Although $~ 80 \%$ maximum size, it possesses a suite of characters associated with immaturity, and is regarded as a subadult. The ornamentation is characterized by a small, recurved, but fused nasal horncore; short, rounded postorbital horncores; and short, triangular, and flat parietal processes. Using this specimen, and additional skulls and bonebed material, the cranial ontogeny of Styracosaurus is described, and compared to Centrosaurus. In early ontogeny, the nasal horncores of Styracosaurus and Centrosaurus are thin, recurved, and unfused, but in the former the recurved morphology is retained into large adult size and the horncore never develops the procurved morphology common in Centrosaurus. The postorbital horncores of Styracosaurus are shorter and more rounded than those of Centrosaurus throughout ontogeny, and show greater resorption later in ontogeny. The length and thickness of the parietal processes increase drastically through ontogeny, but their position and orientation are static across the size series. Several diagnostic Styracosaurus albertensis specimens now preserve medially orientated P3 spikes, causing issues for the diagnosis of S. ovatus. Variability in parietal ornamentation, either expression of P1 and P2 parietal processes, or other cranial ornamentations, does not appear to correlate with stratigraphy.
\end{abstract}

\section{INTRODUCTION}

Styracosaurus is an iconic centrosaurine ceratopsid dinosaur characterized by large, posteriorly and laterally projecting spike-like epiossifications on the posterolateral margins of the parietosquamosal frill (Lambe 1913; Ryan et al. 2007). It is stratigraphically restricted to the Campanian-aged upper Dinosaur Park Formation, 29-50 $\mathrm{m}$ above the contact with the underlying Oldman Formation (Ryan et al. 2007; Brown 2013). With the exception of three fragmentary skulls from the Two Medicine Formation of Montana (Gilmore 1930; McDonald 2011), it is also restricted geographically to the area within, or close to, Dinosaur Provincial Park and Manyberries in southern Alberta. Although first described over 100 years ago (Lambe 1913), few representative specimens were known until relatively recently (Ryan et al. 2007; Holmes et al. 2020). Juvenile skulls of well-represented ceratopsid taxa are relatively rare, and with one notable exception (Currie et al. 2016) are either incomplete (Goodwin et al. 2006; Mallon et al. 2015) or are represented by isolated elements and specimens from monodominant bonebeds (Dodson and Currie 1988; Tokaryk 1997; Ryan 2007). Other putative juvenile ceratopsid material has been described (Gilmore 1917; Gilmore 1922; Dodson 1989), but these specimens have proven difficult to link to diagnostic

Published May 11, 2020

*corresponding author. $\odot 2020$ by the authors; submitted February 18, 2020; revisions received April 22, 2020; accepted April 28, 2020. Handling editor: Jordan Mallon. DOI 10.18435/vamp29361

Vertebrate Anatomy Morphology Palaeontology is an open access journal http://ejournals.library.ualberta.ca/index.php/VAMP Article copyright by the author(s). This open access work is distributed under a Creative

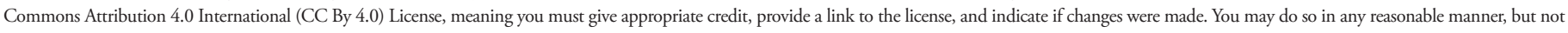
in any way that suggests the licensor endorses you or your use. No additional restrictions - You may not apply legal terms or technological measures that legally restrict others from doing anything the license permits. 
adult material, reducing their utility for studies of ontogeny (but see Penkalski and Dodson 1999; McDonald 2011). Sampson et al. (1997) and Frederickson and TumarkinDeratzian (2014) reviewed the craniofacial ontogeny of Centrosaurinae and Centrosaurus, respectively, but most of the available data for diagnostic material pertains to larger subadults and adults. As such, little is still known about the early ontogeny of the Ceratopsidae, including Styracosaurus. One recently collected Styracosaurus specimen (TMP 2009.080.0001) comprises a nearly complete skull and partial postcranial skeleton. Comparison with other known specimens of Styracosaurus indicates that this specimen is the smallest, and likely youngest, essentially complete skull known for the genus. As such, it provides the opportunity to explore ontogeny and individual variation in this taxon, specifically the development of the cranial ornamentation, and to contrast these patterns with the better sampled close relative Centrosaurus apertus.

\section{MATERIALS AND METHODS}

The specimen TMP 2009.080.0001 was examined at the Royal Tyrrell Museum of Palaeontology. The specimen consists of a nearly complete skull, a posterior cervical vertebra, two dorsal vertebrae, sacrum, two ribs and a scapulocoracoid. Measurements were taken using digital and dial calipers (under $150 \mathrm{~mm}$ ) and fiberglass measuring tape (over $150 \mathrm{~mm}$; Tab. 1). Unless otherwise stated, measurements follow those of Ryan et al (2007). Photographs were taken using a Canon EOS 6D digital SLR camera with 50 $\mathrm{mm}$ [1:1.4] and 24-105 mm [1:4] lenses. Scientific line drawings were prepared from photographs. Dimensions were checked against the specimen, and then the final reconstructions were inked in using Koh-i-noor Rapidograph pens. Photographs were prepared into figures, i.e., removing backgrounds, assembling composite images, using Adobe Photoshop and Illustrator (CS5).

As part of a review of ontogeny and variation in the genus, all available diagnostic Styracosaurus albertensis material, particularly articulated skulls, parietosquamosal frills, and cranial material from monodominant bonebeds was examined. These materials were spread across five museums: Canadian Museum of Nature, Ottawa (CMN), Royal Alberta Museum, Edmonton (RAM), Royal Ontario Museum, Toronto (ROM), Royal Tyrrell Museum of Palaeontology, Drumheller and its Field Station in Dinosaur Provincial Park (TMP), and the University of Alberta Laboratory for Vertebrate Palaeontology, Edmonton (UALVP). One exception is a heavily reconstructed skull at the American Museum of Natural History (AMNH 5372), although additional cranial material (TMP 2006.019.0005) of this specimen including parietal spikes collected in 2006 and 2015 - was examined at the TMP. A full list of specimens is provided in Appendix 1. To quantify variability in the position and orientation of the serially homologous parietal spines, the position and orientation of each spike was measured in each reasonably complete parietal of Styracosaurus. The radial position of the base of each process along the margin of the parietal was measured by projecting a circular coordinate (compass) onto the centre of the fenestra (with $0^{\circ}$ orientated medially, $90^{\circ}$ oriented posteriorly, and $180^{\circ}$ oriented laterally; Appendix 2). A radial coordinate system was chosen because this metric is independent of specimen size and robust to inconsistencies in the shape of the periphery of the parietal. The centre of the fenestra was approximated as the intersection point of the transverse line bisecting the fenestra at the anteroposterior midpoint, and a parasagittal line bisecting the fenestra at the transverse midpoint. Similarly, the orientation of the long axis (base to apex) of each spike was recorded using the same orientations $\left(0^{\circ}=\right.$ medial, $90^{\circ}=$ posterior, and $180^{\circ}=$ lateral). All measurements were taken to the nearest degree using Image (V 1.44) from dorsal images. Resulting data were plotted using R studio (V 3.4.3). In addition to material of Styracosaurus, a large sample of the closely related (Ryan et al. 2012) species Centrosaurus apertus was also examined to provide a comparison with the cranial ontogeny in Styracosaurus. Specifically, an exhaustive sample of nasal horncores and postorbital horncores of Centrosaurus apertus from both articulated skulls and bonebed material, was examined (Appendix 3). A size series (based on basal size measurements) of Centrosaurus and Styracosaurus horncores in lateral views was created used Adobe Illustrator (CS5).

The assumed homologies of epiparietal follow those of Ryan (1992) and Sampson (1993; 1995) with epiossifications being numbered from medial to lateral. Under such a scheme a process projecting from the dorsal margin of the parietal, posterior to the fenestra, and curving anteriorly (if present) is designated $\mathrm{P} 1$, a process projecting from the posterior surface of the parietal and curving medial is designated P2, the medial most posterior spike is designated P3, and so on. For the squamosal, the epiossifications are numbered from anterior to posterior.

\section{GEOLOGICAL AND GEOGRAPHICAL CONTEXT}

The Styracosaurus skull TMP 2009.080.0001 was found in August of 2008 and collected in August of 2009. The specimen was recovered from the upper (southern) portion of the central fork of Princess Coulee, just south of Dinosaur Provincial Park, Alberta (UTM; 12U, 461295 mE, 5617724 mN, 695.75 masl; Fig. 1), in the upper Dinosaur Park Formation (Campanian). The 
Table 1. Measurements of Styracosaurus skull TMP 2009.080.001 following numbering from Ryan et al. 2007. Measurements in mm.

\begin{tabular}{|c|c|c|c|c|}
\hline & Dimension & Median & Left & Right \\
\hline 1 & skull, midline length & 1213 (no rostral) & & \\
\hline 2 & skull, total length & 1435 & & \\
\hline 3 & rostral-orbit length & & 540 & 530 \\
\hline 4 & rostral-posterior margin of nasal horncore & 395 & & \\
\hline 5 & rostral-back of tooth row & & - & - \\
\hline 6 & rostral-epijugal & & 710 & 660 \\
\hline 7 & interorbital width & 320 (rt. Side X2) & & \\
\hline 8 & postorbital HC length & & 51 & 53 \\
\hline 9 & postorbital HC, antpost length & & 70 & 70 \\
\hline 10 & postorbital HC, Mediolateral width & & 40 & 37 \\
\hline 11 & orbit length & & 112 & 108 \\
\hline 12 & orbit, height & & 103 & 98 \\
\hline 13 & orbit, max diameter & & 120 & 126 \\
\hline 14 & post. margin of ext. naris-orbit & & - & 250 \\
\hline 15 & nasal horncore height & 170 & & \\
\hline 16 & nasal horncore basal length & 133 & & \\
\hline 17 & nasal horncore basal width & 65 & & \\
\hline 18 & jugal, minimal width & & 101 & 100 \\
\hline 19 & jugal-orbit length & & - & 238 \\
\hline 20 & lateral temporal fenestra-orbit & & - & 115 \\
\hline 21 & anterior margin of uto-parietal fenestra & & - & 214 \\
\hline 22 & squamosal, min. depth at medial sq. notch & & 196 & - \\
\hline 23 & squamosal caudal length & & 310 & 300 \\
\hline 24 & squamosal, caudal depth & & 270 & 270 \\
\hline 25 & ant. margin of pof. font-back of par. bar & 620 & & \\
\hline 26 & frontal fontanelle length & 180 & & \\
\hline 27 & frontal fontanelle, minimum width & 60 & & \\
\hline 28 & frontal fontanelle, depth & - & & \\
\hline 29 & parietal, total length & 670 & & \\
\hline 30 & parietal, midline bar length & 440 & & \\
\hline 31 & parietal, minimum $1 / 2$ width & & - & 390 \\
\hline 32 & parietal, transv. width of midline bar (post. end) & 148 & & \\
\hline 33 & transv. $1 / 2$ par. width to tip of $\mathrm{P} 4$ & 390 & & \\
\hline 34 & transv. $1 / 2$ par. width to tip of $\mathrm{P} 6$ & & - & 458 \\
\hline 35 & parietal fenestra, max length & & - & 240 \\
\hline 36 & parietal fenestra, max width & & - & 230 \\
\hline 37 & transv. $1 / 2$ par. width to notch between $\mathrm{P} 5$ and $\mathrm{P} 6$ & 390 (as in 31) & & \\
\hline 38 & basal length & 690 (to back of ltc & - Scant & tal., 2014) \\
\hline
\end{tabular}

\begin{tabular}{|c|c|c|c|c|c|c|c|}
\hline Epiossifications & Length & Width & Thickness & Epiossifications & Length & Width & Thickness \\
\hline S1 (left) & 18 & 58 & 12 & P3 (left) & - & 91 & 41 \\
\hline S1 (right) & 31 & 68 & $11^{*}$ & P3 (right) & 135 (18 $\mathrm{mm}$ lost $)$ & 85 & 30 \\
\hline S2 (left) & - & - & 19 & P4 (left) & - & 114 & 29 \\
\hline S2 (right) & 20 & 58 & - & P4 (right) & 130 & 75 & 30 \\
\hline S3 (left) & $29^{*}$ & 73 & 18 & P5 (left) & - & - & - \\
\hline S3 (right) & 22 & 62 & - & P5 (right) & 73 & 69 & 20 \\
\hline S4 (left) & 21 & 69 & 15 & P6 (left) & - & 66 & 17 \\
\hline S4 (right) & 23 & 55 & - & P6 (right) & 27 (from suture) & 41 & 15 \\
\hline S5 (left) & - & - & - & P7 (left) & 18 & 69 & 13 \\
\hline S5 (right) & & & & P7 (right) & 17 & 20 & 8 \\
\hline P1 (left) & - & - & 27 & P8 (right) & 12 & 50 & 8 \\
\hline P1 (right) & 26 & 83 & 34 & & & & \\
\hline P2 (left) & - & - & 25 & \multirow{2}{*}{\multicolumn{4}{|c|}{$*_{\text {indicates best estimate }}$}} \\
\hline P2 (right) & - & - & - & & & & \\
\hline
\end{tabular}




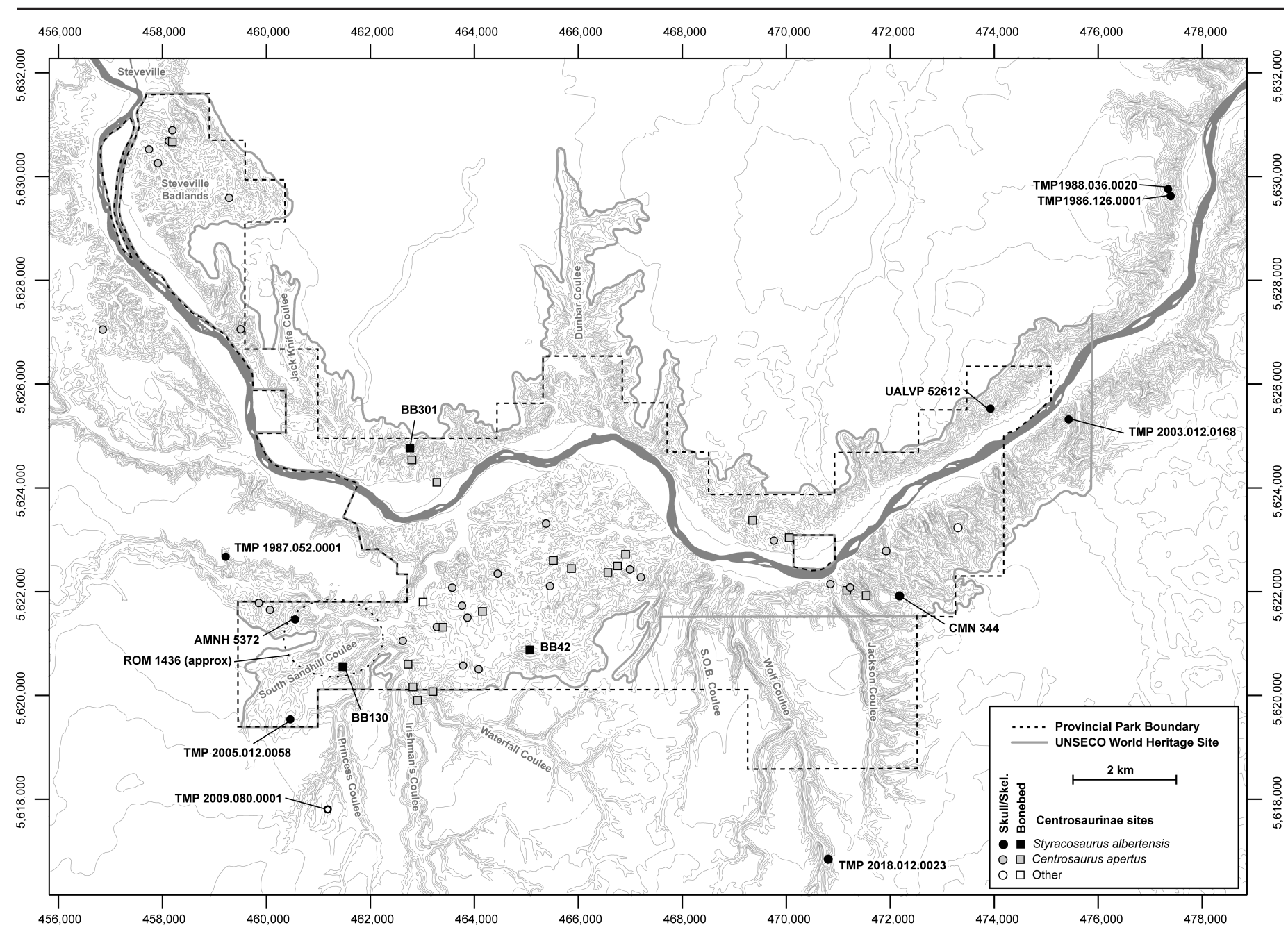

Figure 1. Map of Dinosaur Provincial Park, Alberta, illustrating the geographic localities of significant centrosaurine ceratopsid sites (quarries and bonebeds). Styracosaurus albertensis sites (black) are specifically labelled, while those of Centrosaurus apertus (grey) and other centrosaurines (white) (e.g., Coronosaurus brinkmani, 'c.f. Pachyrhinosaurus') are unlabelled. Circles indicate skulls and/or skeletons while squares indicate bonebeds. Hollow black circle is TMP 2009.080.0001. TMP 1989.097.0001 and UAVLP 55900 are located outside of the mapped area. UTM Coordinates (Zone 12U) are indicated in the margin.

geographic position of the specimen in the upper reaches of the coulee system, just below prairie level, is similar to several other Styracosaurus albertensis specimens including AMNH 5372 and TMP 1987.052.0001 (Sandhill Creek), TMP 2005.012.0058 (South Sandhill Coulee) and TMP 2018.012.0023 (Wolf Coulee), or in the eastern portion of the main Red Deer River Valley (CMN 344, TMP 1986.126.0001, TMP 1988.036.0020, UALVP 52612), all of which represent outcrops of upper levels of the Dinosaur Park Formation. This is in contrast to the location of the majority of the Centrosaurus apertus quarries, which are found low in section within the Dinosaur Park Formation (Fig. 2), and are concentrated in the Core, Steveville Badlands, and main Red Deer River Valley (also known as Dead Lodge Canyon; Fig. 1).

The specimen was found at the base of a $4.5 \mathrm{~m}$ thick palaeochannel, $48 \mathrm{~m}$ above the contact between the Dinosaur Park Formation and underlying Oldman
Formation (Fig. 2). The host sandstone is characterized by inclined bedded sandstone with large to medium-scale trough crossbedding, carbonaceous drapes and a basal lag of ironstone clasts and fossils. The host sandstone overlies an organic rich mudstone and is in turn overlain by a marker shale (700.25 masl). The stratigraphic position of the specimen falls within the previously documented Styracosaurus zone (Ryan et al. 2007), but occurs fairly high in this zone, being one of the highest measured specimens in the Park area.

The specimen is a partial, disarticulated skeleton, with an articulated and nearly complete skull. The skull was positioned ventral side up with the left side of the parietal exposed to the midline, and the left squamosal eroding out of the rock. Several fragmented ribs were also exposed on the surface. The glenoid of the displaced scapulocoracoid was found hooked around the nasal horncore. 


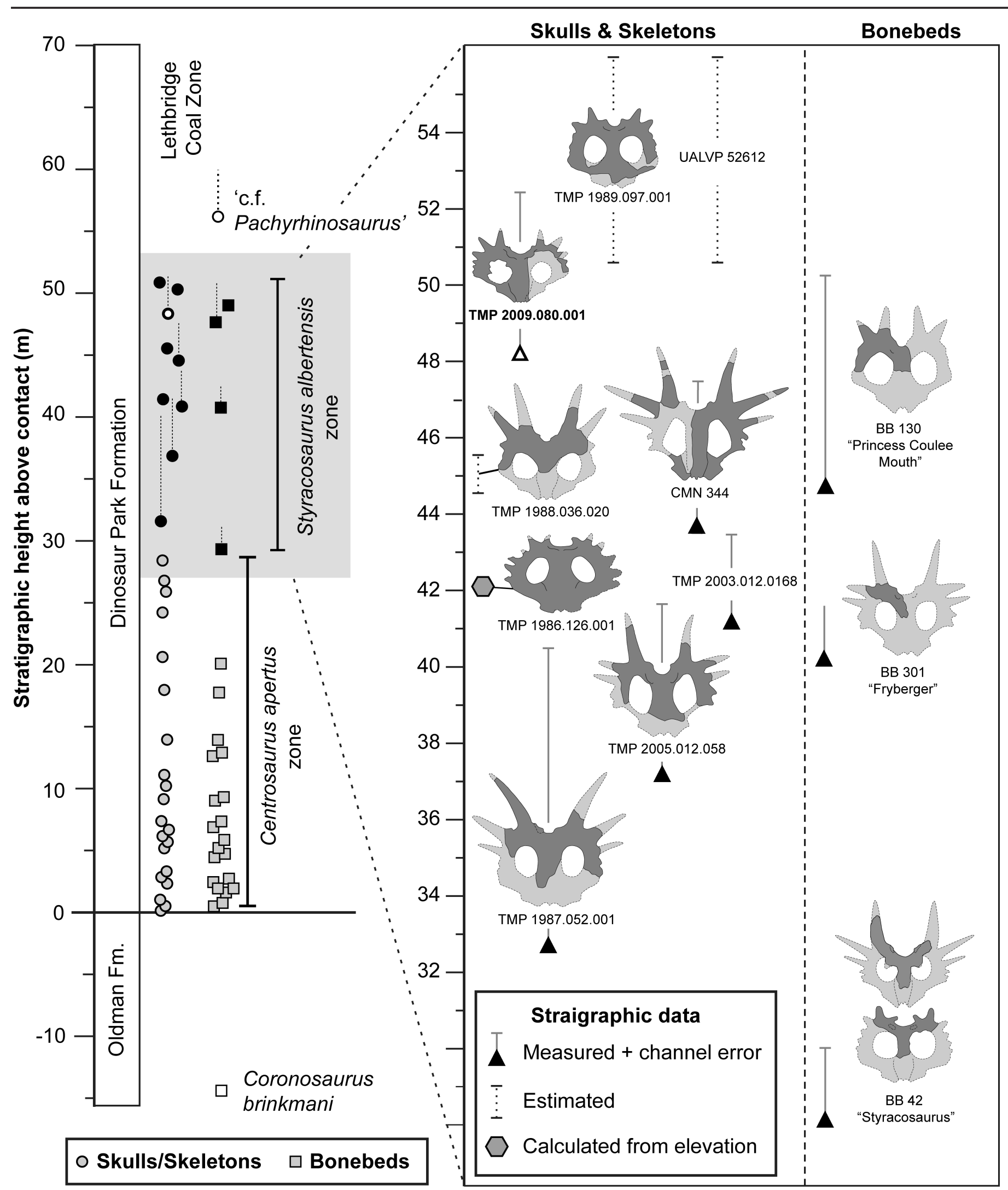

Figure 2. Stratigraphic position of Styracosaurus albertensis specimens within the Dinosaur Park Formation (Campanian) of Alberta, including both bonebeds and isolated skulls and skeletons. The column on the left shows the stratigraphic position of significant centrosaurine specimens, while the inset on the right highlights the parietal morphology of individual Styracosaurus specimens. Bonebeds samples show representative diagnostic specimen only (bottom to top: TMP 1999.055.0005, 1984.093.0001, 2016.015.0005, 2016.012.0029). Stratigraphic position is based on height above the Dinosaur Park/Oldman formation contact. Stratigraphic data derived from Brown (2013). Hollow circle and triangle indicate TMP 2009.080.0001. 


\section{DESCRIPTION}

The skull of TMP 2009.080.0001 has been prepared to expose the dorsal and lateral aspects; the ventral aspect is obscured by the supporting plaster jacket (Figs. 3-5). It is nearly complete with only the rostral bone and lower jaws missing. The associated right quadrate and quadratojugal became dissociated during burial (Fig. 6A, B), and were found appressed to the lateral surface of the squamosal; they have since been removed from the main skull block. The left half of the frill is broken into several pieces, but the lateral margins of both the left squamosal and parietal appear to be nearly complete and the bases of the parietal spikes are preserved in place (Figs. 3B, 5).

The palate and braincase are not exposed. However, the midline distance between the tip of the snout (missing the rostral) and transverse line drawn between the posterior margins of the lateral temporal openings, a proxy for basal length (Scannella et al. 2014), is $690 \mathrm{~mm}$. Adding an additional $50 \mathrm{~mm}$ to accommodate the missing rostral still makes this individual, with an estimated basal length of $740 \mathrm{~mm}$, the smallest nearly complete Styracosaurus skull known.

The right side of the skull is more complete and better preserved, although the squamosal has broken into two pieces and telescoped, and the dental ramus of the maxilla is obscured or more likely not preserved. Dorsoventral crushing of the skull has resulted in lateral splaying of the left half of the skull relative to the right, and as a result, the left side of the nasal bearing the posterior narial margin has rotated up and is visible in dorsal view (Fig. 3B). The left cheek, orbital region, and interorbital region have drifted as a unit a short distance laterally, and have rotated into the frontal plane. The jugal and squamosal are both broken, although the region of the lateral temporal opening is preserved.

\section{Snout}

Premaxilla: The snout is relatively longer than in the holotype specimen (CMN 344). As a result, the nasal vestibule is distinctly longer than it is tall (Figs. 3A, 4A). Dorsally, the premaxilla is clasped by anterior processes of the nasal. The anterior margin of the premaxilla has broken away and has moved upward slightly. Otherwise, the snout is well-preserved and complete. The interpremaxillary suture had not fused in this region at the time of death (Fig. $4 \mathrm{~B})$. Although a dorsal portion of the internarial septum has broken away and become slightly displaced, it is still possible to determine the shapes and sizes of the septum and narial vestibules. As in other centrosaurines, the ventral (oral) margin of the premaxilla is strongly convex (Figs. 3A, $4 \mathrm{~A})$. The posteroventral process of the premaxilla is broad and spatulate with no posterior bifurcation. It reaches the lacrimal posteriorly, preventing the maxilla from making contact with the nasal. This condition is atypical for most centrosaurines, but appears to be variable in Centrosaurus, and may be similarly variable in Styracosaurus.

Maxilla: Only the dorsal portion of the right maxilla is preserved; the inset, tooth-bearing ramus is obscured. It has slid slightly anteroventrally, exposing its underlapping surface with the jugal and displacing the trough-like anterior extension of the antorbital foramen ventrally. No tooth count or tooth row length can be determined.

Nasal: Anteriorly, the bifurcated anterior process of the nasal clasps the median posterodorsal process of the premaxilla (Fig. 3B). Immediately posterior to these premaxillary processes, the dorsal surface of the nasal is swollen and rugose and bears prominent grooves. Posterior to this, the nasal bears a pointed and distinctly posteriorly recurved horncore $170 \mathrm{~mm}$ tall (Figs. 3A, 4A). This thin and recurved morphology has been identified as juvenile morphology in Centrosaurus (Sampson et al. 1997; Frederickson and Tumarkin-Deratzian 2014). The lateral surface of the horncore is covered in distinct, largely longitudinal anastomosing grooves, the largest of these measuring $-140 \mathrm{~mm}$ long ( $82 \%$ total height) and $5 \mathrm{~mm}$ wide. The horncore is laterally compressed and blade-like, such that the cross-section is an elongate oval with the minor (transverse) axis about $40 \%$ that of the major (anteroposterior) axis. This cross section is maintained along the height of the horncore, from the base (134 mm long, 55 $\mathrm{mm}$ wide) to the mid-height ( $85 \mathrm{~mm}$ long, $38 \mathrm{~mm}$ wide). Distinct, sharp ridges characterize the anterior and posterior extremes of the horncore along much of this length, but these become gradually rounded in the basal quarter. Despite its small size, the horncore shows no obvious external midline sutures, suggesting the left and right sides of the horncore are largely fused. This condition is also exhibited by a nasal horncore of nearly identical size and morphology (TMP 2009.031.0001; Fig. 7-specimen 55) collected from BB 301 (Fryberger Styracosaurus bonebed). In overall form, it also closely resembles the morphology of a very small, isolated, and unfused presumably juvenile horncore (TMP 1998.093.0163; Fig. 7-specimen 54; Ryan et al. 2007:fig. 8E, F, note this is incorrectly reported as TMP 1994.014.0866 in that caption), although it is more than twice the size (Tab. 1).

The nasal horncore is situated posterodorsal to the nasal vestibule, with most of the horncore posterior to, but the anteriormost third overlapping with, the nasal vestibule. Ventral to the horncore, the nasal forms the posterior margin of the nasal vestibule. The nasal appears to contribute to the septum, but a suture with the premaxilla cannot be identified. The narial process projecting from the posterior rim of the vestibule is distinctly inflected anteriorly into the vestibule. 


\section{Circumorbital region}

The two orbits are noticeably different in shape; the left orbit is nearly square with rounded corners (Fig. 5), and the right is distinctly longer measured along its posterodorsal-anteroventral axis than it is measured dorsoventrally (Figs. 3A, 4A) and oddly, the dimensions of the left and right orbits are quite similar (Tab. 1, 11-13). Not all of this apparent difference can be accounted for by post-mortem distortion, indicating that there must have been some asymmetry in orbit shape during life. The orbits are relatively large, at 15 percent (average of all orbit dimensions) of basal skull length (Figs. 3A, 4A). Comparative relative
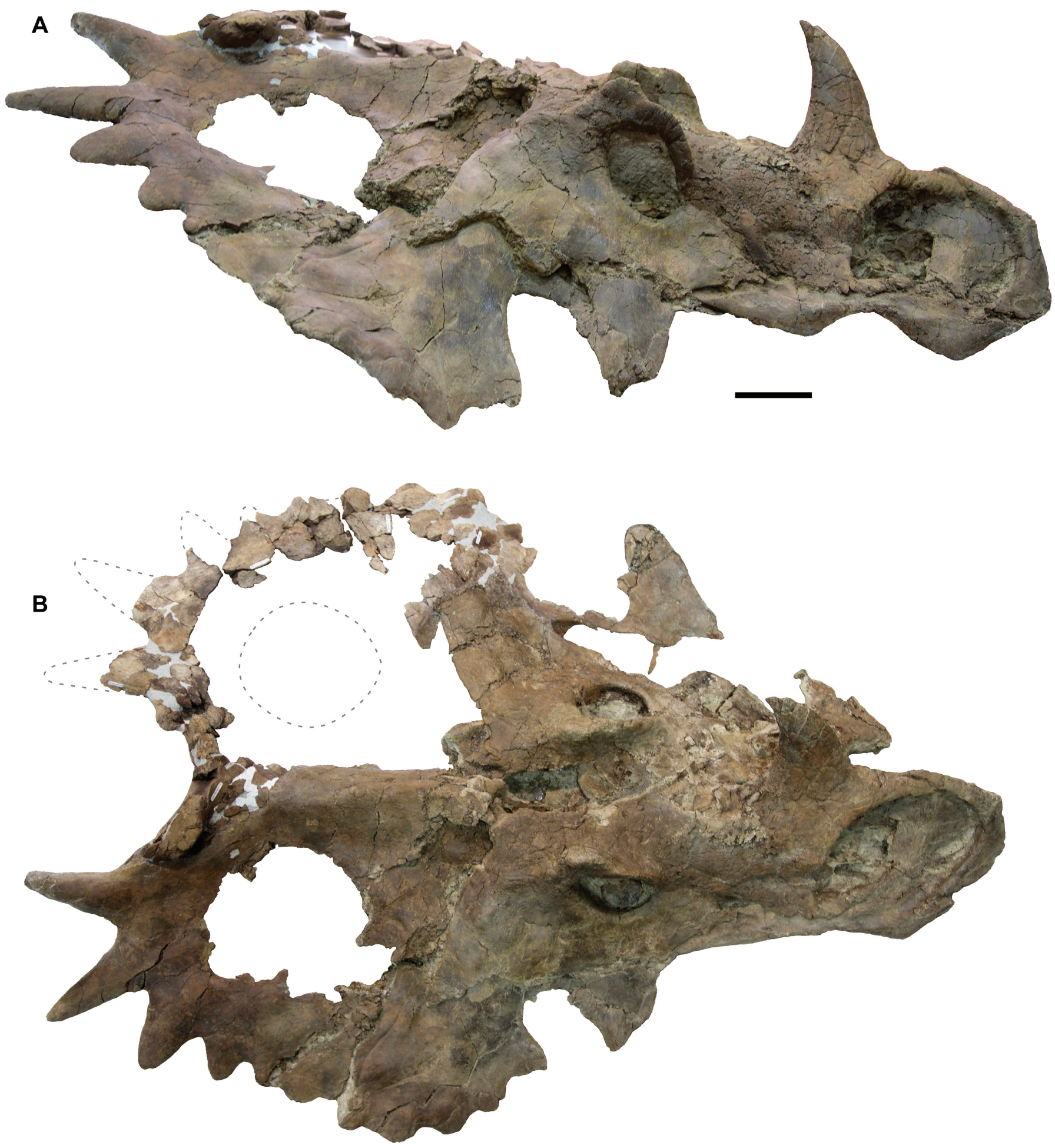

Figure 3. Right lateral (A) and dorsal (B) photographs of TMP 2009.080.0001, a subadult skull of the centrosaurine ceratopsid Styracosaurus albertensis. Dashed lines represent approximate extent of the left side of parietal. Scale bar $=10 \mathrm{~cm}$. 
orbit lengths for known, and presumed adult, Centrosaurus specimens average $13.5 \%(\mathrm{SD}=0.02, \mathrm{n}=14)$.
Palpebral: The palpebral forms the curved anterodorsal and dorsal margins of the orbit. It is well integrated into

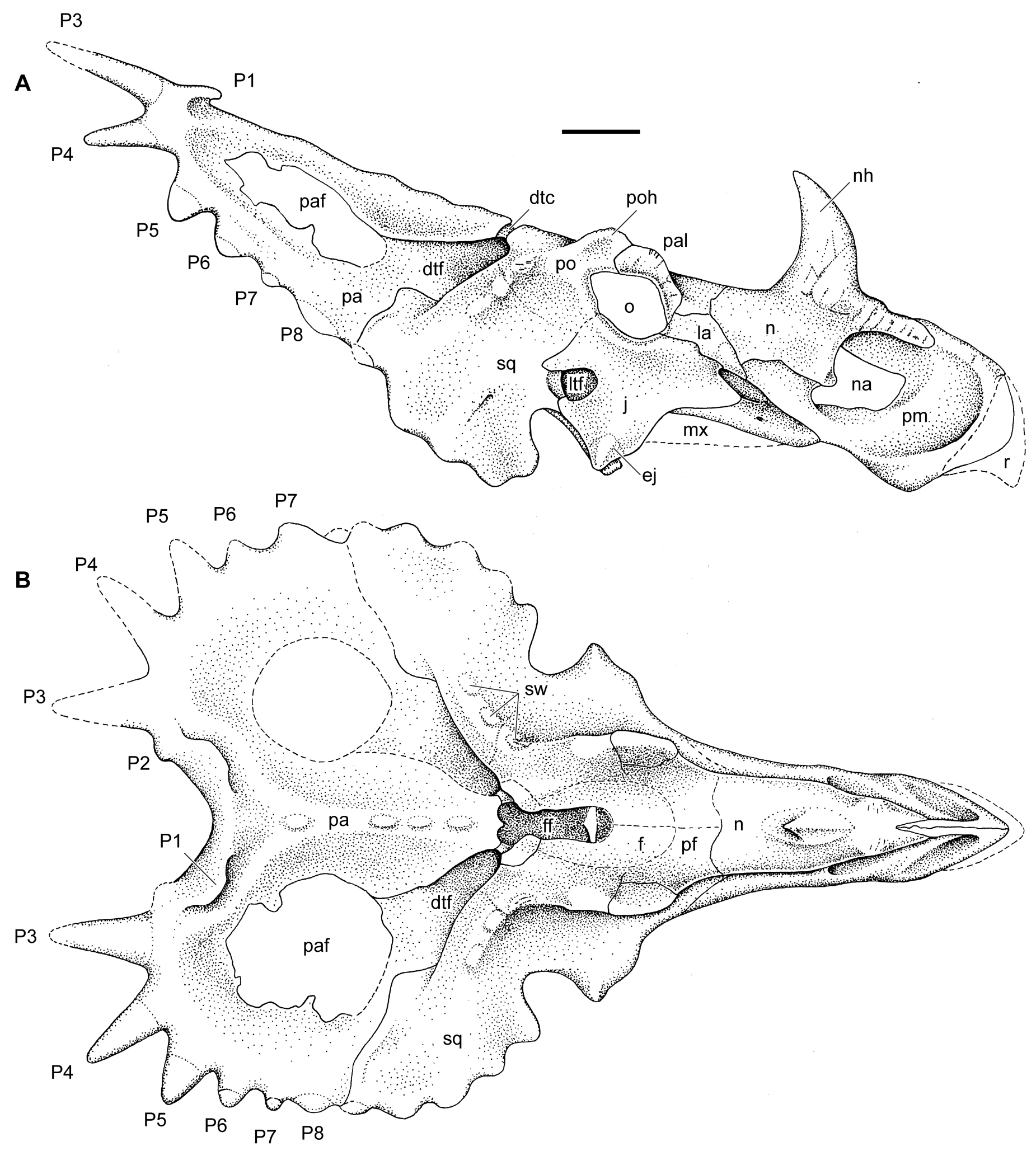

Figure 4. Right lateral (A) and dorsal (B) line drawings of TMP 2009.080.0001, a subadult skull of the centrosaurine ceratopsid Styracosaurus albertensis. Scale bar $=10 \mathrm{~cm}$. Abbreviations: ej, epijugal (or facet); dtf, dorsotemportal fenestra; dtc, dorsotemportal channel; f, frontal; ff, frontoparietal fontanelle; j, jugal; la, lacrimal; Itf, laterotempotal fenestra; mx, maxilla; n, nasal; na, nasal ventibule; nh, nasal horncore; o, orbit; P\#, parietal process; pa, parietal; paf, parietal fenestra; pal, palpebral; pf, prefontal; pm, premaxilla; po, postorbital; poh, postorbital horncore; sq, squamosal; sw, swelling. 
the skull, but traces of its sutures with the postorbital and prefrontal are still visible (Fig. 4). It bears a well-developed antorbital buttress with subtle radially oriented grooves on its flattened lateral face.

Lacrimal: The lacrimal is well integrated into the skull, but its sutures with the surrounding bones can be traced (Fig. 4A). It is elongated with a triangular anterior process. In contrast with most ceratopsids, the antorbital buttress of the palpebral does not extend ventrally onto the lateral surface of the lacrimal.

Prefrontal, Frontal: The sutures between the frontals, prefrontals and nasals are quite well coossified, but the remaining traces allow their approximate courses to be reconstructed (Fig. 4B). Together, the frontal and prefrontal form the smooth, slightly arched skull roof from the posterior margin of the nasal to the anterior margin of the
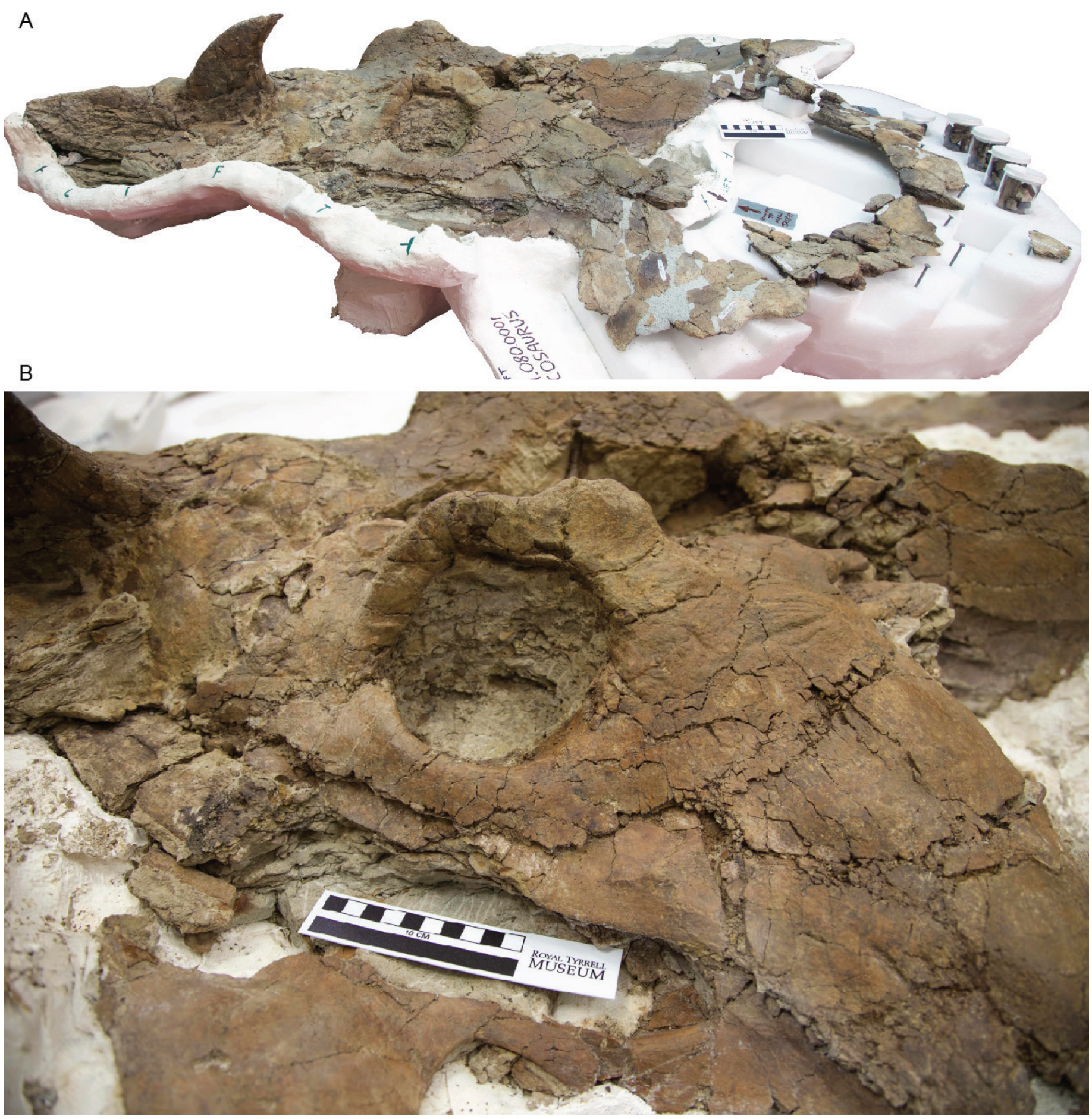

Figure 5. Photographs of the left side of TMP 2009.080.0001, a subadult of Styracosaurus albertensis, showing the overall skull (A) and an inset of orbital region (B). Scale bars $=10 \mathrm{~cm}$. 

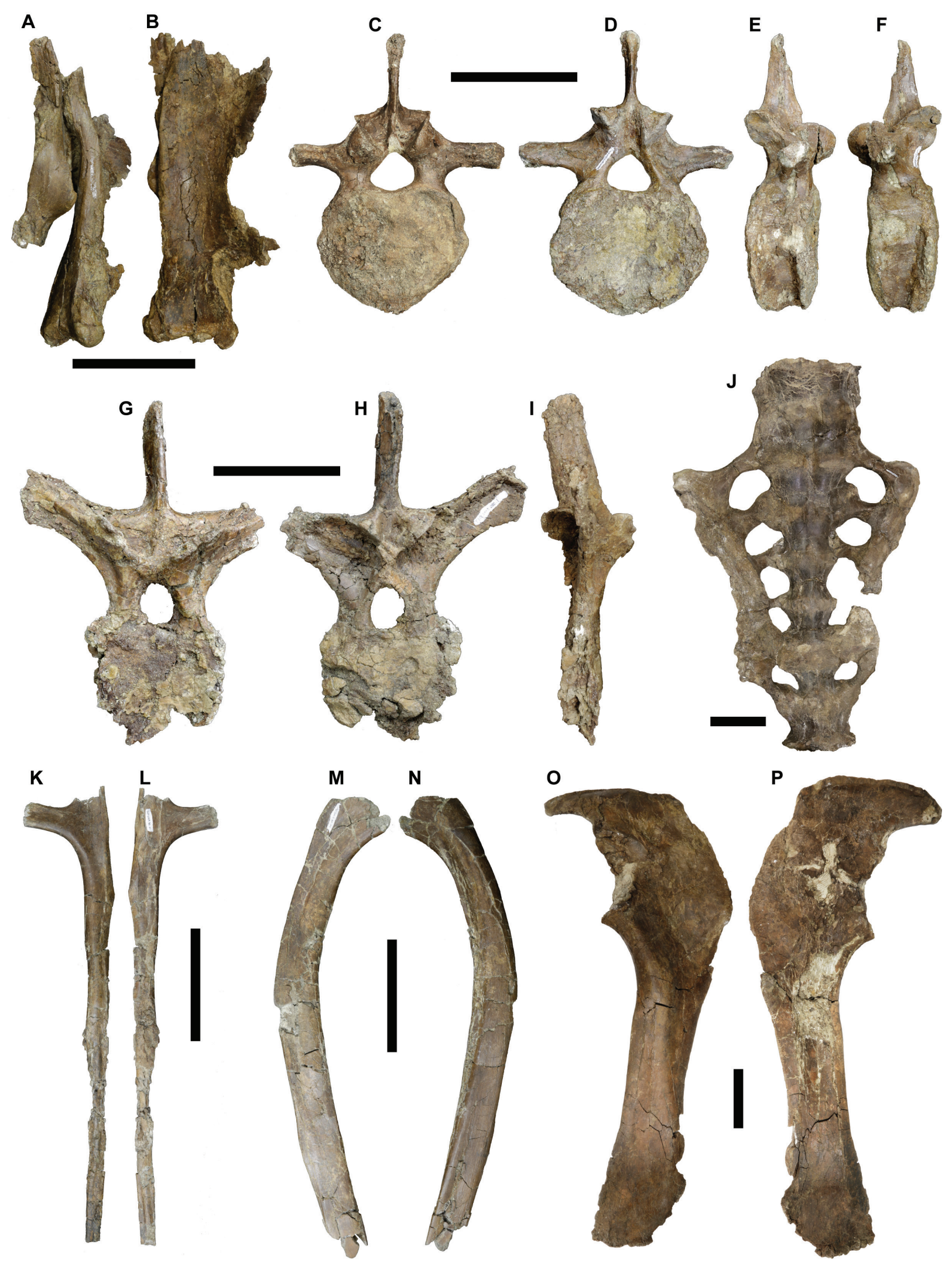

Figure 6. Photographs of associated cranial and postcranial element of TMP 2009.080.0001. A \& B, associated right quadrate and quadratojugal in lateral $(A)$ and anterior (B) views. $C-F$, cervical vertebrate $(C 5)$ in anterior (C), posterior (D), right lateral (E), and left laterial $(F)$ views. G-I, dorsal vertebrate in anterior $(\mathrm{G})$, posterior $(\mathrm{H})$ and left lateral $(\mathrm{I})$ views. J, sacrum in ventral view. K-L, posterior left cervical rib in anterior $(\mathrm{K})$ and posterior $(\mathrm{L})$ views. $\mathrm{M}-\mathrm{N}$, partial left dorsal rib in posterior $(\mathrm{M})$ and anterior $(\mathrm{N})$ views. $\mathrm{O}-\mathrm{P}$, left scapulocoracoid in lateral $(\mathrm{O})$ and medial $(\mathrm{P})$ views. All scale bars $=10 \mathrm{~cm}$. A-I \& K-N, and J \& O-P are at the same scale. 


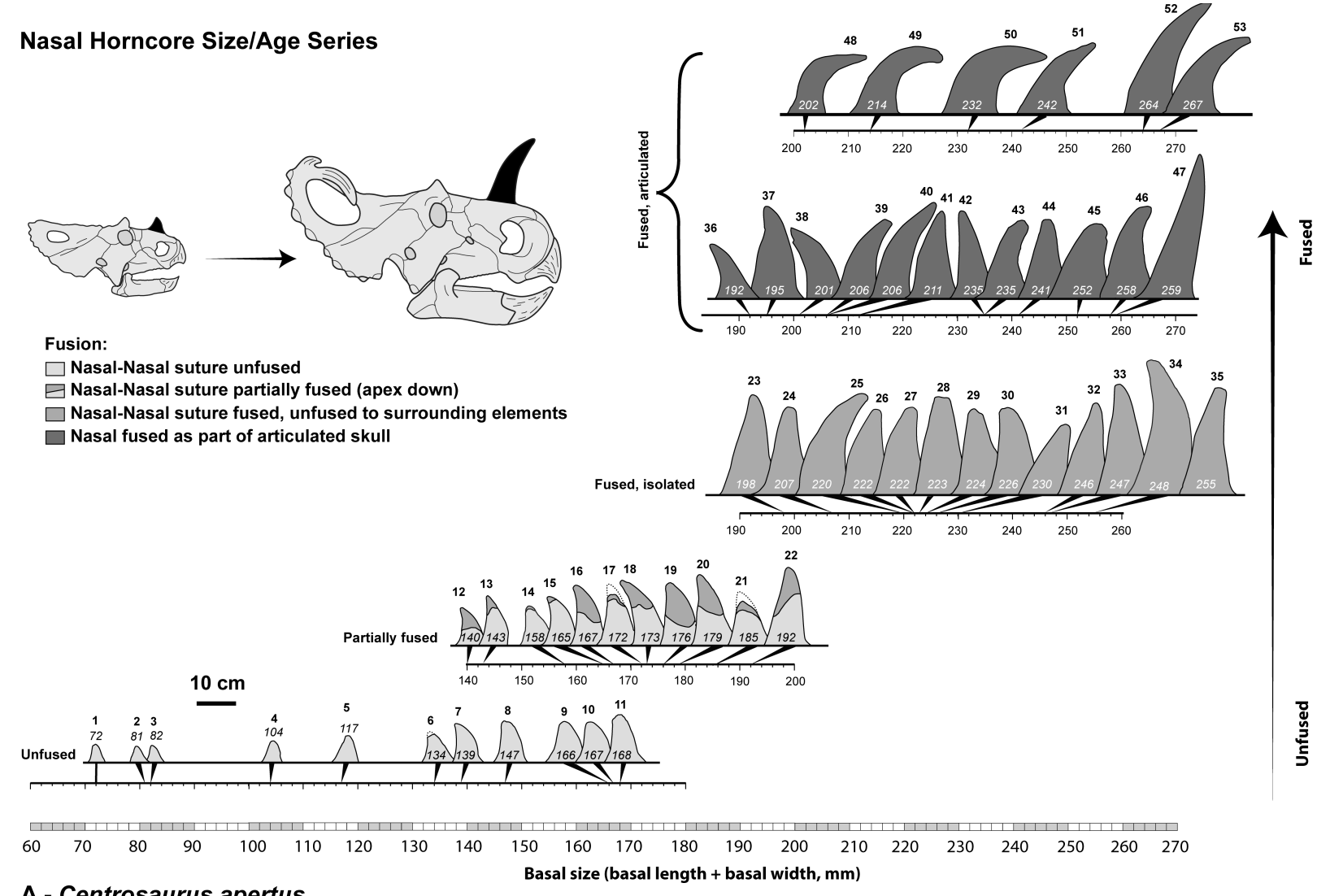

\section{A - Centrosaurus apertus}

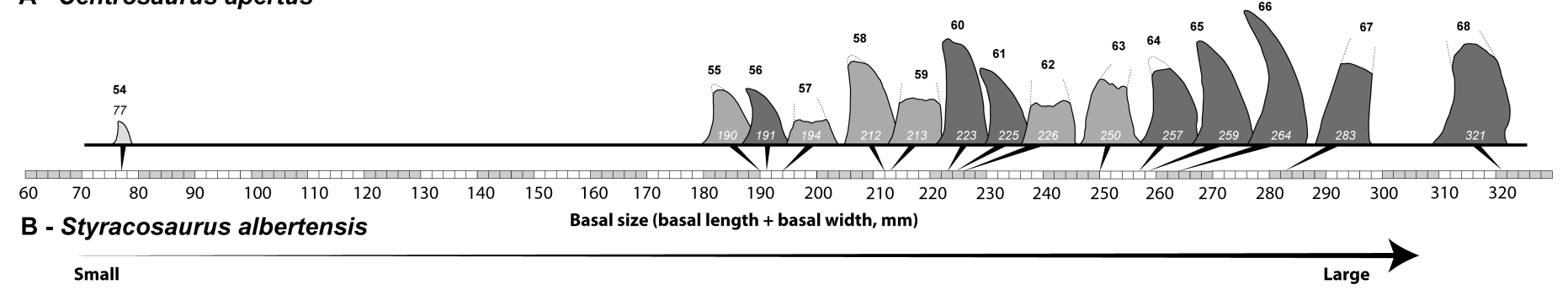

Figure 7. Size series of Centrosaurus apertus (A, upper) and Styracosaurus albertensis (B, lower) nasal horncores in right lateral view. Specimens are arranged along the horizontal axis according to their size (basal length plus basal width), and those of Centrosaurus are separated based on their degree of cranial sutural fusion (shaded infill). Although ordered based on size, the absolute position does not correlate directly with size due to spacing constraints. Numbers in italics indicate basal size metric, while numbers in bold refer to specimen numbers listed in Appendix 3.

frontoparietal fontanelle. Laterally they are bounded by the lacrimal, palpebral, and postorbital.

Postorbital: The postorbital forms the posterodorsal and posterior margins of the orbit and a short section of the anterior margin of the dorsotemporal opening. Immediately anterior to the latter opening, the postorbital bears a low, knob-like swelling traversed by anteroposteriorly oriented grooves (Fig. 4). A similar low knob is also seen in another partial Styracosaurus skull (TMP 2003.012.0168). The postorbital-squamosal suture appears not to have coossified at the time of death, at least on the better-preserved right side. The postorbital horncore is short $(-50 \mathrm{~mm}$, apex to orbital rim) and rounded dorsally with a slightly concave lateral surface resulting in an overall reniform morphology in dorsal view, being $-70 \mathrm{~mm}$ long and $-40 \mathrm{~mm}$ wide (Figs. 3, 4). Its morphology is very similar to other individuals of comparable size/age both from Styracosaurus bonebeds (e.g., TMP 1966.010.0041; Fig. 8-specimen 105) and partial skulls (e.g., TMP 1986.126.0001). It bears some general similarity to smaller horncores described for juvenile Centrosaurus (Sampson et al. 1997; Ryan et al. 2007), but is quite unlike the tall, pointed and distinctly three-sided pyramidal horncores commonly present in Centrosaurus specimens of similar size. In contrast to other known Styracosaurus skulls (with the exception of TMP 1986.126.0001), the horncores show no distinct evidence of resorption pits. The apices of both horncores, however, bear small roughened patches that may represent incipient 


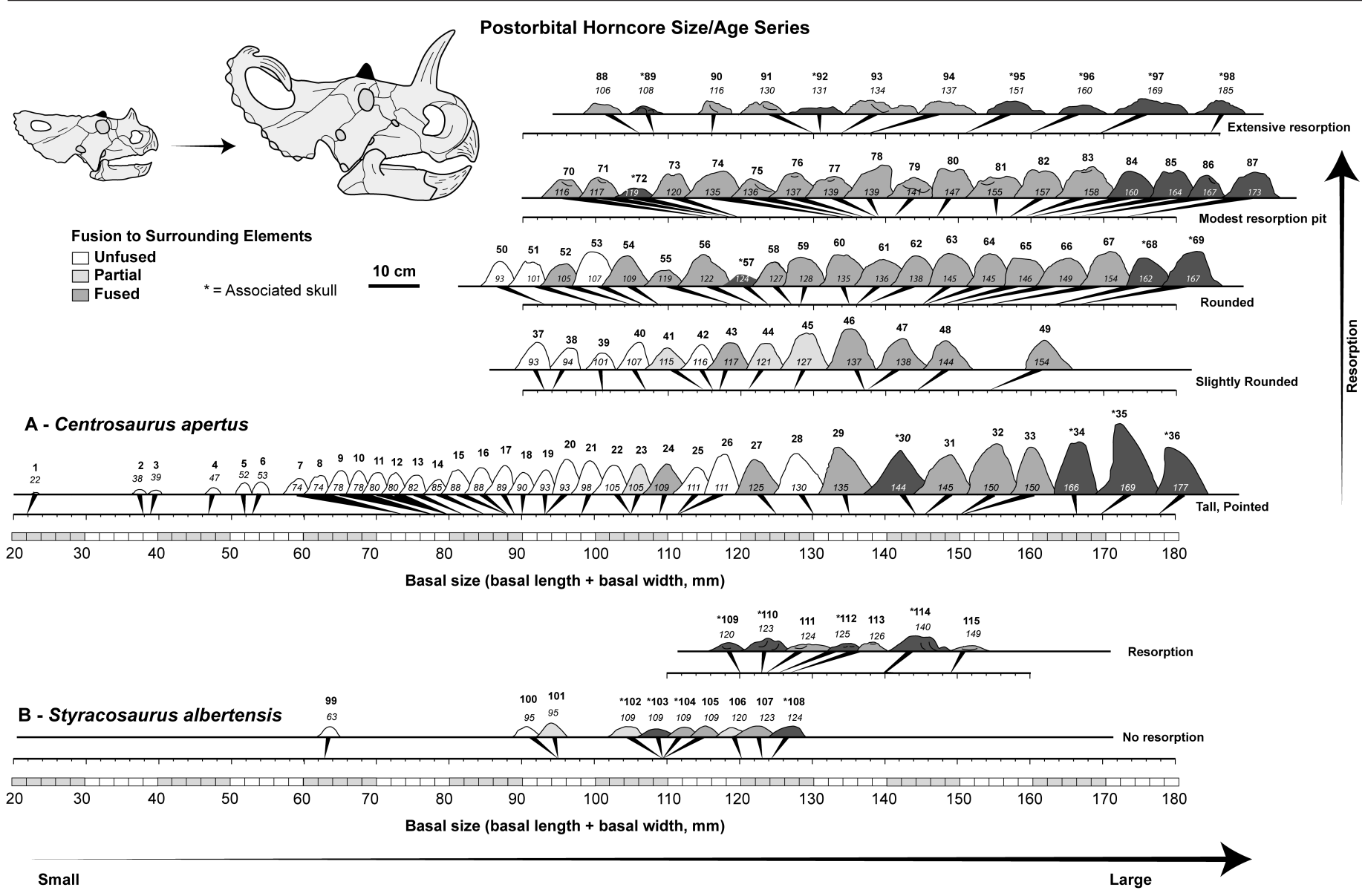

Figure 8. Size series of Centrosaurus apertus ( $\mathrm{A}$ - upper) and Styracosaurus albertensis (B - lower) postorbital horncores in right lateral view. Specimens are arranged along the horizontal axis according to their size (basal length plus basal width), and separated vertically based on their degree of resorption. Although ordered based on size, the absolute position does not correlate directly with size due to spacing constraints. Numbers in italics indicate basal size metric, while numbers in bold refer to specimen numbers listed in Appendix 3.

resorption, or taphonomic damage, but lack the conspicuous longitudinal furrows and coarsely rugose texture seen in adult specimens of Centrosaurus and Styracosaurus.

Jugal: Both jugals are preserved. The right jugal overlaps the maxilla to form an oblique anterodorsally trending suture that extends far anteriorly. As a result, the jugal forms the superficial ventral margin of the antorbital foramen (Figs. $3 \mathrm{~A}, 4 \mathrm{~A})$. This feature is unusual for centrosaurines, in which the jugal-maxillary suture is usually more vertical, and the jugal does not reach as far forward. This is variable in expression in Centrosaurus. Posterior to its suture with the maxilla, the jugal sweeps ventrally, and at its ventral tip swells laterally into a vertically inclined ridge bearing distinct rugosities. It is unclear if this swelling represents a fused, modestly sized epijugal ossification, or is merely the facet for one, the latter being more likely. This ambiguous anatomical interpretation makes it impossible to determine the precise size or shape of the epijugal. Disruption and telescoping of the right cheek have obscured the lateral temporal opening on that side, although its ventral margin is preserved. The left jugal pre- serves the dorsal, anterior, and ventral margins of a large (approximately $54 \mathrm{~mm}$ in vertical dimension) lateral temporal opening. The bone has broken in two along a horizontal line about $30 \mathrm{~mm}$ below the orbit, and the ventral part has slid ventrolaterally about $50 \mathrm{~mm}$. As a consequence, the jugal has become separated from its overlapping sutural connections with the squamosal immediately above and below the lateral temporal opening (Figs. 3B, 5).

Quadrate/quadratojugal: The nearly complete right quadrate and complete right quadratojugal are slightly disarticulated from each other, with the quadratojugal displaced posteromedial and dorsal relative to the quadrate, such that it is positioned posterior to the quadrate (Fig. 6A, B). The two elements had drifted away from their natural position onto the dorsal surface of the skull and were removed during preparation. The quadrate is missing its dorsal extremity, but the thin medial pterygoid wing is largely complete. This wing has a smaller ventrally positioned triangular projection, and a larger dorsally positioned rounded projection. Laterally the quadrate forms a rugose 
butt-suture for the displaced quadratojugal, the ventral extreme of which is flattened and expanded into a triangular anterior process. Ventrally the quadrate swells to form the spool-like condyle. The medal and lateral swellings of the quadrate condyle are nearly equal in size and shape but the medial condyle extends slightly further ventrally. The displaced quadratojugal is wedge shaped, with broad, flattened sutural contact for the quadrate posteromedially and jugal anterolaterally. Only a thin wedge of quadratojugal would be exposed on the exterior surface of the skull. A triangular swelling projects from the posterolateral margin of the quadratojugal, and would be confluent with the swollen ventral margin of the jugal.

Frontoparietal Fontanelle: The right margin of the frontoparietal fontanelle appears to be complete except toward its anterior end (Figs. 3B, 4B). It is impossible to determine the exact size of the fontanelle because of the disruption and displacement on the left side of the skull, but appears to have been large, approximately $50 \mathrm{~mm}$ across at its widest point, and about $170 \mathrm{~mm}$ long. Near the anterior end of the fontanelle, the right side of a well-developed transverse buttress (Sternberg 1927) extends to the midline. The anterior extent of the fontanelle lies between the two postorbital horns. The floor of the fontanelle was shattered and largely lost post-mortem, so its original depth cannot be estimated with any confidence.

\section{Frill}

Squamosal: Both squamosals are preserved, but only the right is complete (Fig. 3B). However, at some point during preservation, the latter broke into a dorsal portion (still articulated with the jugal and postorbital) and a ventral portion, and the latter then slid dorsomedially between 35-50 $\mathrm{mm}$ under the dorsal portion. A short process projects anteriorly below the lateral temporal fenestra to form a suture with the jugal, excluding the quadratojugal from forming any part of the ventral margin of the opening. The left squamosal bears the thickened, deeply bevelled posterior margin of the lateral temporal opening. Anterodorsal and anteroventral to the opening are the underlapping articular facets for the displaced jugal. The posterior part of the left squamosal is broken into several pieces, but its lateral edge appears to be almost complete. It bears a minimum of four episquamosal loci, although space is available along the poorly-preserved posterolateral aspect for a fifth; five may be a more accurate count. The more complete right squamosal bears four scalloped and gently convex episquamosal loci, but distinct episquamosals appear to be absent. A fifth epiossification straddles the lateral end of the squamosal-parietal suture (Figs. 3, 4). The main body of the squamosal is flat to slightly convex. Two low swellings are arranged diagonally across the element. More dorsally, two low swellings parallel the anterior margin of the dorsotemporal opening and align with a third swelling on the postorbital.

Parietal: The parietal still retains its connections with both postorbitals, although the bridge underlying the left dorsotemporal channel that connects the frontoparietal fontanelle with the left dorsotemporal fenestra (Farke 2010) fractured and separated as the distal head of the median bar rotated counter clockwise about 25 degrees. The median, anterior projection of the median bar is preserved (Figs. 3B, 4B). In both its overall morphology, and the frill ornamentation, the parietal of TMP 2009.080.0001 is most similar to that of another small Styracosaurus specimen TMP 1987.097.0001. The median bar is wide, with slightly concave dorsolaterally facing sides rising steeply to form a wedge-shaped median ridge. A prominent median ridge is characteristic of protoceratopsids. A similar morphology is also present in TMP 1986.126.0001, another small skull, but is not seen in larger Styracosaurus skulls, where the bar is broadly rounded dorsally. The same ontogenetic trends have been noted in Centrosaurus (Sampson et al. 1997; Frederickson and Tumarkin-Deratzian 2014) and chasmosaurines (Currie et al. 2016). Posterior to the midpoint of the bar, the median ridge becomes more gently rounded. The ridge bears three prominent lenticular knobs anteriorly, and a slightly less conspicuous knob more posteriorly - these are also much less prominent in larger skulls (e.g., CMN 344, UALVP 55900). The right parietal fenestra is roughly oval in shape, with the long axis oriented anterolaterally (Figs. 3B, 4B). Its margin is highly irregular. It is unlikely that this simply reflects damage and loss of bone, as the right half of the frill is otherwise well preserved. It is more likely that, as in UALVP 55900 (Holmes et al. 2020), the bone had not ossified sufficiently to form a smoothly curved fenestral border. The lateral fenestral margin is interrupted by a distinct triangular wedge of bone (35 $\mathrm{mm}$ long) projecting from the lateral parietal bar into the centre of the fenestra. This morphology is seen on some Centrosaurus specimens (e.g., CMN 8798), but other than UALVP 55900 (Holmes et al. 2020) has not been reported in Styracosaurus.

Both P1 epiossifications are preserved, but the right, upon which the following description is based, is much more complete (Figs. 3B, 4B). It is broad-based but short. Unlike the holotype (CMN 344), it is distinctly procurved. However, unlike some Styracosaurus specimens (Ryan et al. 2007; Holmes et al. 2020: figs. 9, 14), it is squared off in dorsal view rather than triangular. Immediately anterior to its base, the dorsal surface of the parietal bears a shallow sulcus. The left P2 is relatively prominent, but is triangular to tab-like in outline rather than hook-like as in ROM 1436, TMP 1986.126.0001 (Ryan et al. 2007:figs. 13, 14), and UALVP 55900 (Holmes et al. 2020). The right P2 is not 
preserved, but its base can be identified immediately medial to the right P3. Six additional epiparietals are preserved on the right parietal (Figs. 3B, 4B). Parietal processes at P3 and $\mathrm{P} 4$ are straight, triangular, spike-like, and are slightly dorsoventrally compressed with oval cross-sections. The apex of P3 is incomplete; based on the shape of the preserved portion, about $18 \mathrm{~mm}$ of its tip is missing, making it the longest of the series (Tab. 1). Unlike the holotype, it projects posteriorly and slightly medially. The complete, posterolaterally projecting P4 is slightly shorter than the estimated total length of P3, but otherwise of similar morphology. Processes at loci $\mathrm{P} 5$ and $\mathrm{P} 6$ are also triangular, but progressively shorter than P4, and more dorsoventrally compressed, with P5 being approximately as long as wide. Epiparietal 7 is more knoblike and much less well coossified with the parietal than the other epiparietals. The process at P8 is fully ' $\mathrm{D}$ '-shaped in outline, and indistinguishable from the homologous ossification of Centrosaurus. The left parietal is not as well preserved, but appears to be similar to the right side. The lateral parietal processes on the left are similar in morphology to those on the right, with a few minor exceptions. On the left side, the base of $\mathrm{P} 4$ is larger than that of $\mathrm{P} 3$, the reverse of the situation on the right. In addition, the epiossification count on the left is less than the right, with only seven loci. It is uncertain whether a transitional ossification spanning the parietal-squamosal suture was present. This suggests at least some asymmetry in the parietal ornamentation. Unlike some centrosaurines (e.g., Sinoceratops - Zhucheng Dinosaur Museum V0010, Wendiceratops - TMP 2011.051.0009, 2014.029.0097), none of the lateral epiossifications curve dorsally/anteriorly. As in the holotype, their bases are in the plane of the frill, and distally curve slightly ventrally. There is little or no indication of imbrication.

\section{Postcranial skeleton}

\section{Vertebrae: Specimen TMP 2009.080.0001 includes} three presacral vertebrae and the sacrum. The best preserved presacral vertebra is a posterior cervical (Fig. 6C-F). The anterior and posterior facet of the centrum is slightly heartshaped (Fig. 6C), with a distinct parapophysis positioned on its lateral-most surface, slightly dorsal to the midpoint (Fig. 6E, F). Its centrum is $114 \mathrm{~mm}$ in transverse diameter (width), $50 \mathrm{~mm}$ in length, and $91 \mathrm{~mm}$ in vertical diameter (height). The neural canal is relatively large ( $31 \mathrm{~mm}$ wide, $29 \mathrm{~mm}$ tall) and a rounded triangle in shape, with the apex directed dorsally. The transverse processes are directed laterally in the horizontal plane and are in line with the dorsal extent of the neural canal. The triangular, dorsally tapering neural spine is $53 \mathrm{~mm}$ tall, orientated vertically, and has a transverse expansion at its dorsal apex. The pre- and postzygapophyses arise from the anterior and posterior bases of the neural spine dorsal to the transverse processes. A thin midline ridge, a ventral continuation of the neural spine, separates the prezygapophyses, while a reciprocal midline notch separates the postzygapophyses. The oval postzygapophyseal facets lie at $-45^{\circ}$ to the horizontal, while those of the prezygapophyses are steeper at $-60^{\circ}$. In morphology, this vertebra closely matches the fifth, or possibly sixth, cervical vertebra (second and third free cervicals) in the holotype of Styracosaurus (CMN 344: Holmes and Ryan 2013). The total height of the vertebra is $210 \mathrm{~mm}$, and the width between transverse processes is $158 \mathrm{~mm}$. While the centrum width is of similar size to the homologous element in the holotype specimen (CMN 344), other measures are smaller (Holmes and Ryan 2013). Neurocentral synchondroses are fused on this and all other known vertebrae of the specimen. A posterior trunk vertebra is more poorly preserved, with its centrum badly crushed and much of its external surfaces lost (Fig. 6G-I). As a result, it is difficult to determine its dimensions. No distinct parapophysis is observed, but given the condition of the bone this may be obscured. The preserved portion of the centrum appears to be oval in anterior and posterior views, taller than wide, with a preserved transverse diameter (width) of $99 \mathrm{~mm}$, and vertical diameter (height) that is incomplete. Due to crushing, the centrum length cannot be determined. The neural canal is oval in shape, $25 \mathrm{~mm}$ wide and $32 \mathrm{~mm}$ tall. The transverse processes project dorsolaterally at an angle of $-45^{\circ}$ to the horizontal, and are only slightly posteriorly inclined. Although the left process is incomplete, the transverse distance between the apices would have been $-200 \mathrm{~mm}$. The prezygapophyses are positioned in line with the base of the transverse processes, while the postzygapophyses are located further dorsally. The circular to oval facets of both the pre- and postzygapophyses lay at $-45^{\circ}$ to the horizontal. The neural spine is tall $(84 \mathrm{~mm})$, rectangular, and slightly posteriorly inclined. The total height of the vertebra is estimated at $-254 \mathrm{~mm}$.

A second dorsal vertebra is also present, but it is poorly preserved with most of its surface lost, and cannot be identified with confidence. Both the centrum and the neural canal are taller than wide, and the arch is taller and more constricted than the previously described dorsal. Further details cannot be determined.

The nearly complete synsacrum has been described previously (Holmes and Ryan 2013). It is prepared in its field jacket, such that only the ventral surface is exposed (Fig. $8 \mathrm{~J})$. The synsacrum is composed of an ankylosed series of two dorsosacrals, four true sacrals and two caudosacrals with a total length of $662 \mathrm{~mm}$. The dorsosacral centra have the greatest transverse widths, with the anterior of the two measuring $149 \mathrm{~mm}$, and the posterior $169 \mathrm{~mm}$. In contrast, the first sacral centrum is an isosceles trapezoid, with a broad anterior width $(140 \mathrm{~mm})$ and greatly constricted 
posterior width $(104 \mathrm{~mm})$. The remaining sacral centra are narrow (85-67 $\mathrm{mm}$ in an anteroposterior sequence) and spool shaped. The second caudosacral is transversely wider $(109 \mathrm{~mm})$ than the first $(70 \mathrm{~mm})$. The ventral surfaces of the two dorsosacrals and first two sacrals are relatively flat, with a shallow medial groove, whereas the centra of the remaining sacrals and first caudosacral form a continuous midventral keel. The synsacrum supports six broad based sacral ribs, the anteriormost anchoring from the posterolateral margin of the last dorsosacral and the anterolateral margin of the first sacral (S1), and the posteriormost anchoring from the posterolateral margin of the first caudosacral and anterolateral margin of the second caudosacral. Laterally the sacral ribs expand anteroposteriorly to form a contiguous, crescentic acetabular buttress and iliac facet $381 \mathrm{~mm}$ long. The anteriormost sacral rib is the longest $(172 \mathrm{~mm})$ whereas the lengths of the ribs gradually decrease to the fourth $(87 \mathrm{~mm})$, and increase again to the sixth $(112 \mathrm{~mm})$.

Ribs: Two left ribs are preserved. One has a straight shaft and widely separated proximal articulations (although the parapophysis is largely missing) identifying it as a posterior cervical (Fig. 6K, L). The shaft, missing only the distal extremity, measures $396 \mathrm{~mm}$ in preserved length. It is largely circular in cross-section, with a midpoint diameter of 19 $\mathrm{mm}$. The other rib is larger, with a flat, curved shaft, and lacks the proximal end (Fig. 6M, N). However, its large size identifies it as an anterior trunk rib. It is oval in cross-section with an anteroposterior diameter of $39 \mathrm{~mm}$, and a transverse diameter of $13 \mathrm{~mm}$.

Scapulacorocoid: A complete left scapulocoracoid is preserved (Fig. 6O, P), and was closely associated with the skull - as preserved the glenoid was hooked around the nasal horncore. The entire unit is $798 \mathrm{~mm}$ long, with the scapula being $663 \mathrm{~mm}$ long. The greatest width of the scapula is $212 \mathrm{~mm}$, while the minimum width, located at midshaft, is $102 \mathrm{~mm}$. The two elements are well fused, such that the dorsal portion of their suture is difficult to demarcate. The coracoid is $-305 \mathrm{~mm}$ long and $174 \mathrm{~mm}$ in maximum width, and bears a distinct coracoid foramen, the fossa of which is $-30 \mathrm{~mm}$ in diameter. The coracoid is characterized by a crescentic anterior margin, which shows a distinct medial folding, a ventral margin with a hook-like ventral process (90 mm long) anteriorly and glenoid fossa posterior, and a relatively straight posterior margin fused to the scapula. The glenoid fossa is $145 \mathrm{~mm}$ long, $65 \mathrm{~mm}$ in maximum thickness, and nearly equally bisected by the scapulocoracoid suture, with the scapula contributing only slightly more to the glenoid than the coracoid. The shaft of the scapula is rectangular with a distinct dorsoventral expansion at its anterior, and to a lesser extent, posterior extremes. The lateral surface of the scapula is dominated by the round scapular spine, which traverses from the anteroventral margin (posterior margin of the glenoid) diagonally across the element to flatten out near the posterodorsal margin. Medially, the scapula is flat. The posterior margin of the scapula is incomplete, but square, and only slightly expanded in thickness.

In both size and morphology, the scapulocoracoid closely matches that of TMP 1989.098.0001, another small Styracosaurus, with both being $-85 \%$ the length of the holotype (CMN 344). In contrast with the larger holotype, the two smaller specimens (TMP 2009.080.0001 and 1989.098.0001) show a less distinct and less swollen margin around the glenoid fossa, a less developed anterior crescentic margin of the coracoid, and posterior region of the scapular shaft that is less expanded.

\section{DISCUSSION}

Immature status of TMP 2009.080.0001. Several size-independent features on the skull suggest that TMP 2009.080.0001 is ontogenetically young and not representative of the standard adult Styracosaurus condition. Many of these features are consistent with known patterns of craniofacial ontogeny in Centrosaurinae (Sampson et al. 1997) and Centrosaurus (Frederickson and Tumarkin-Deratzian 2014), and some have been documented previously for Styracosaurus (Ryan et al. 2007). The midline interpremaxillary suture is open in anterior view (Frederickson and Tumarkin-Deratzian 2014, char. 12:0). The nasal horncore is small (char. 15:0), laterally compressed and blade-like (char. 17:0), and is recurved (char. 13:0). The palpebral-postorbital suture is only partially obscured (char. $41: 1$ ). The postorbital horncore is low and rounded (char. 37:0), lacks any indication of resorption pits (char. 32:0), and is not dominated by a rugose texture (char. 36:0). All squamosal epiossification sutures are open (in fact, no squamosal epiossifications are present) (char. 48-51:0). Although the parietal fenestrae fall above the $50 \%$ sagittal length threshold for the juvenile character state in (Frederickson and Tumarkin-Deratzian 2014, char. 58:1), they are relatively small. The posterior and lateral parietal bars are thin (char. 72:0).

In addition to the ontogenetic characters identified by Frederickson and Tumarkin-Deratzian (2014), there are several other features indicating the specimen may be a young animal. The median bar of the parietal is triangular in cross section, and bears a pronounced, acuminate dorsal median ridge, similar to TMP 1986.126.0001 but in contrast with larger specimens where this ridge is broadly rounded. Additionally, the lateral epiossification loci are inline (i.e., not imbricated), the orbit is relatively large (orbit size is negatively allometric in vertebrates), and the antorbital buttress of the palpebral does not extend onto the lacrimal. 
Although the skull of TMP 2009.080.0001 exhibits many immature features, distinct long-grain and mottled bone textures (Frederickson and Tumarkin-Deratzian, 2014; char. 9, 30, 56, 80), which are good size independent indicators of relatively young age in Centrosaurinae (Sampson et al. 1997; Brown et al. 2009; Tumarkin-Deratzian 2010), are entirely absent. Rather, the surficial bone texture is more consistent with a subtle adult texture. This absence of longgrained and mottled bone textures is not easily attributed to taphonomic damage as the specimen shows distinct bone textures elsewhere, and other bonebed derived specimens show these distinct textures even when the elements have been subject to higher degrees of abrasion and damage. Nevertheless, the short, triangular, and dorsoventrally compressed morphology of P3 and P4 in TMP 2009.080.0001 closely matches that of other, presumed subadult material of Styracosaurus, both from BB42 (e.g., TMP 1981.019.0223, TMP 1995.055.0005) and isolated skulls (e.g., TMP 1997.012.0122), all of which do show long-grained and mottled textures. This suggests TMP 2009.080.0001 may only just have lost the mottled textures seen on these specimens. The loss of these textures in Centrosaurus is correlated with the development of the parietal ornamentation (Brown et al. 2009), so their absence in this skull, with small but diagnostic ornamentation is not surprising.

Based on the suite of character states, it is likely the specimen had not achieved skeletal maturity. However, the skull, with a basal length of about $740 \mathrm{~mm}$, is relatively large, $-90 \%$ the length of the holotype (CMN 344) and $-80 \%$ the length of the recently described UAVLP 55900. Similarly, the scapula of TMP 2009.080.0001 is $663 \mathrm{~mm}$ long, $-85 \%$ the size of CMN 344, and nearly identical in size to TMP 1989.097.0001 (Fig. 9). Following the definition of Sampson et al. (1997), the specimen is therefore referred to as a subadult, as opposed to a juvenile, because it has attained a body size approaching that of large, adult specimens, but retains several immature features.

Despite its relatively large size, the cranial ornaments are poorly developed. The nasal horncore is less than one-third the height of the holotype specimen (CMN 344-reconstructed), while the parietal processes $\mathrm{P} 3, \mathrm{P} 4$ and $\mathrm{P} 5$, are all only slightly greater than one-quarter their counterparts in the holotype. A nearly identical pattern is seen in TMP 1989.097.0001, which, based on postcranial measurements is $-80 \%$ the size of the holotype (see Holmes and Ryan 2013), yet bears parietal processes approximately $-25 \%$ of the size of those of the holotype (Fig. 9). Together these results suggest that the sizes/expression of the cranial ornaments in Styracosaurus are strongly developmentally delayed, and disconnected from overall skeletal size, such that they do not develop until the individual is near adult size. These results are consistent with previous hypotheses regarding the timing of development of cranial ornaments in Centrosaurinae (Sampson et al. 1997), as well as Hadrosauridae (Evans 2007; Evans 2010; though see Farke et al. 2013), and are consistent with the interpretation of these features evolving in the context of sociosexual selection.

\section{Ontogeny of Cranial Ornaments in Styracosaurus, and Comparison with Centrosaurus. Although} many of the ontogenetic characters well established for Centrosaurinae and Centrosaurus (Sampson et al. 1997; Frederickson and Tumarkin-Deratzian 2014) appear to be reflected in the ontogeny of Styracosaurus, until recently the restricted sample size in the latter made it difficult to evaluate this impression. The recent discovery of several new specimens, in particular TMP 2009.080.0001, now allows for direct comparison between the two taxa, specifically with respect to development of the cranial ornaments.

Nasal: Figure 7 depicts a size series, and hypothesized growth series (although size is not an exact proxy of age), of both Centrosaurus and Styracosaurus nasal horncores constructed using an extensive sample of both articulated skulls and bonebed material. In Centrosaurus, nasal horncore ontogeny progresses from small, equilateral, and unfused forms (Fig. 7, specimens 1-5), through distinctly posteriorly recurved forms with fusion from the apex towards the base (specimens 8-22), into large, fully-fused forms ranging from straight (specimens 23-35), to slightly (specimens 36-47), and strongly (specimens 48-53) anteriorly procurved (Fig. 7A). While exceptions exist (e.g., specimens 36-TMP 1992.082.0001, 38-CMN 8798) and variability in the size/shape at fusion is seen (e.g., specimens 9, 10, 11 vs. 12,13 ), this pattern is both fairly constrained and largely correlated with basal sizes of the horncores and estimated sizes of the individuals.

In Styracosaurus the pattern is similar (Fig. 7B), but with several notable differences. At the smallest size (specimens 1-TMP 1995.400.0074 for Centrosaurus, 54-TMP 1998.093.0163 for Styracosaurus) the two taxa cannot be differentiated, but potential differences are seen as the nasals begin to fuse. Despite the many immature features of the nasal horncore in TMP 2009.080.0001, the nasal-nasal suture in the horncore appears to be fully fused (char. 20:3 of Frederickson and Tumarkin-Deratzian 2014). This is in contrast to the pattern in Centrosaurus, where specimens of similar small size and thin, recurved morphology are generally only partially fused (Fig. 6). TMP 2009.080.0001 (specimen 56) is not unique, as a nearly identical morphology is preserved in a nasal from a Styracosaurus bonebed (specimen 55-TMP 2009.031.0001). Together, these suggest that the paired nasals of Styracosaurus may have fused at a smaller/younger stage than in Centrosaurus. Also, in contrast with Centrosaurus, fused nasals of Styracosaurus largely retain the juvenile condition of being posteriorly 


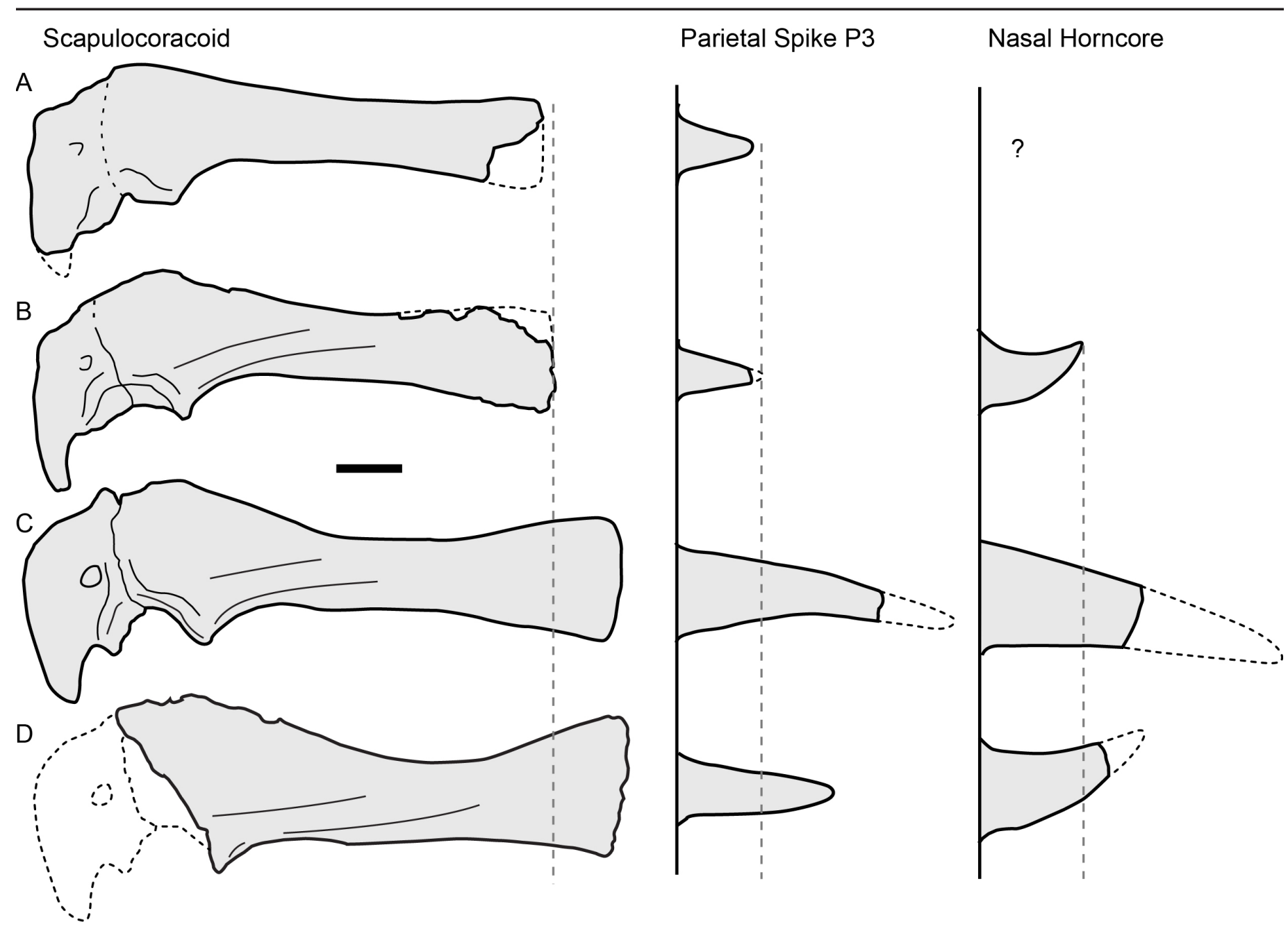

Figure 9. Absolute size comparison between the scapulocoracoid in lateral view (left column), parietal spike $\mathrm{P}_{3}$ in dorsal view (central column), and nasal horncore in left lateral view (right column) for four Styracosaurus specimens. A, TMP 1989.097.0001 (subadult). B, TMP 2009.080.0001 (subadult). C, CMN 344 (adult). D, UALVP 55900 (adult). All elements scaled to the same size. Scale bar $=10 \mathrm{~cm}$.

recurved or, at the most, straight (specimen 67-CMN 344) regardless of the absolute size of the horncore. In this way Styracosaurus horncore morphology mirrors an early ontogenetic stage of that of Centrosaurus, and may represent an example of paedomorphosis, despite the apparent early fusion of the element. Whether or not the fusion at a smaller/earlier growth stage is related to the less variable curvature of the nasal horncore in adults is unclear, but it remains a possibility. As the procurved morphology in Centrosaurus is restricted to fully fused nasals, and largely associated with articulated skulls, it may be that early fusion in Styracosaurus constrains this morphology.

Despite the possibility that horncore shape in Styracosaurus is paedomorphic relative to Centrosaurus, the maximum size of Styracosaurus nasal horncores greatly exceeds that of Centrosaurus (Fig. 7). This represents an interesting juxtaposition in horncore shape versus horncore size between these two closely related animals, where Styracosaurus adults have the nasal horncore morphology of juvenile/subadult Centrosaurus, but are much larger in absolute size.
Postorbital: Figure 8, constructed using both articulated skulls and bonebed material, represents a size series, and hypothesized growth series, of both Centrosaurus and Styracosaurus postorbital horncores. In Centrosaurus postorbital horncore ontogeny progresses from small, laterally compressed hemispherical horncores (Fig. 8, specimens 1-6) to tall, three-sided, and pointed-apex horncores that remain unfused to the surrounding elements (specimens 7-22; Fig. 8A). Fusion with the palpebral and associated elements is accompanied or followed by resorption of the horncore, from slight (specimens 37-49) to increasing (specimens 50-69) roundedness of the apex, development of a distinct resorption pit (specimens 70-87), and nearly complete resorption (specimens 88-98). Several exceptions to this pattern exist, with some horncores showing no resorption, and retaining a tall, pointed-apex morphology to large size (specimens 29-36). There is also a great deal of variation across the size series with respect to where/when fusion and resorption occurred. 
The postorbital horncores of Styracosaurus are distinct from Centrosaurus, even at the smallest comparable sizes (Fig. 8B). The smallest Styracosaurus postorbital horncore (specimen 99-TMP 2014.15.0084) is a low rounded dome, longer than tall. This is in sharp contrast to horncores with similarly sized bases in Centrosaurus (e.g., specimens 7-TMP1979.011.0117 and 8-TMP 1981.022.0013), which are twice as tall and have pointed apices. Postorbital horncores consistently remain short through the entire size series in Styracosaurus, with no specimen developing the tall pointed morphology, regardless of size (Fig. 8B). The postorbital horncores of TMP 2009.080.0001 (specimen 102) differ from the classic 'pyramidal' horncores representative of subadult Centrosaurus (e.g., specimens 22-TMP 1982.018.0017, 23-TMP2015.059.0023, 24-TMP1998.093.0034) in that they are low (half the height of similarly sized horncores in Centrosaurus) with rounded apices and concave lateral surfaces. This morphology is not unique to TMP 2009.080.0001, but is seen in several other bonebed and articulated specimens (e.g., specimens 103-TMP 1986.126.0001, 104-TMP1966.010.0041). At the extreme end of the size series, Styracosaurus does show extensive resorption (e.g., specimens 109-115) similar to Centrosaurus (specimens 89-98), with a complete lack of discrete horncores in many cases. At this stage, the two taxa are largely indistinguishable. One potential difference, however, is that these resorbed horncores of Styracosaurus have smaller basal sizes than in Centrosaurus, another indication that the postorbital horncores in Styracosaurus are truncated, as they do not reach as large a size as in Centrosaurus. A second distinction, based on the current sample, is that a majority of fused Styracosaurus postorbitals show extensively resorbed conditions. This is in contrast to the Centrosaurus sample in which fewer than a third show this condition. It is unclear if these two distinctions are due to sampling differences, taphonomic bias, as a result of Styracosaurus postorbital horncores being absolutely smaller (i.e., having less to resorb), or being resorbed at an earlier stage relative to Centrosaurus.

It is worth noting the apparent reciprocal ontogenetic trajectories between the nasal and postorbital horncores. Relative to Centrosaurus, Styracosaurus nasals are larger but exhibit a juvenile morphology, while Styracosaurus postorbitals are generally smaller and show higher levels of resorption.

Parietal: Figure 10 shows the variation, both ontogenetic and likely independent of ontogeny, across all known relatively complete parietals of Styracosaurus.

Parietal morphology does change dramatically through ontogeny, with those features showing the most dramatic changes being the sizes and shapes of the parietal processes, specifically their apical-basal lengths, dorsoventral thicknesses, and basal circumferences (Fig. 10). Smaller, presumably subadult individuals (e.g., TMP 1989.098.0001 and TMP 2009.080.0001) bear short, thin (dorsoventrally compressed) and triangular parietal processes, while larger, presumably more mature skulls (e.g., CMN 344, TMP 1987.052.0001, 2005.012.0058, ROM 1436, and UALVP 55900) bear thick, rounded in cross section and spike-like parietal processes (Fig. 10). The correlation between parietal process development and absolute size of the frill, however, is imperfect. The most extreme exception to this pattern is TMP 1989.126.0001, which, based on several factors represents a sub-adult specimen, and bears unfused parietal processes of similar shape to other subadult specimens (e.g., TMP 1989.098.0001 and TMP 2009.080.0001). Despite its apparent young age, the parietal of this specimen greatly exceeds many adult specimens (e.g., CMN 344, TMP 1988.036.0020, TMP 2005.012.0058) in most size metrics. This indicates a discontinuity between apparent developmental age and size that may further confuse the understanding of ceratopsid ontogeny. This is consistent with a similar discontinuity seen in extant taxa (e.g., Petermann et al. 2017), and is related to high developmental variation in dinosaurs (Griffin and Nesbitt 2016).

Although Styracosaurus parietal processes transform greatly in absolute size late in ontogeny (Fig. 10), their relative sizes appear fixed early in ontogeny. In nearly all skulls, whether adult or subadult (TMP 2009.080.0001 and TMP 1989.098.0001), P3 is the longest, most massive spike, followed by P4, P5, and P6 respectively (UALVP 55900 in an exception), although the processes are relatively much smaller in the subadults. This suggest that it is possible to recognize and diagnose subadult specimens of Styracosaurus; although identification of younger specimens is likely more problematic. Once again TMP 1989.126.0001 represents an exception to an otherwise consistent pattern. The parietal processes, rather than being heteromorphic with $\mathrm{P} 3$ dominating followed by $\mathrm{P} 4$ and $\mathrm{P} 5$, are all similar in size and morphology (Fig. 10).

The absence of reasonably complete juvenile Styracosaurus parietals and the highly species-specific morphology of the parietal ornamentation (specifically the loci) of Centrosaurus (i.e., char. 59-71, 74-77 of Frederickson and Tumarkin-Deratzian 2014) limit our ability to make detailed comparisons of parietal ontogeny between Centrosaurus and Styracosaurus, but some basic patterns may exist. The median undulations along the midline bar are thought to become more pronounced through ontogeny in Centrosaurus (Frederickson and TumarkinDeratzian 2014). In contrast, in TMP 2009.080.0001 these are more developed than in many larger Styracosaurus 
A
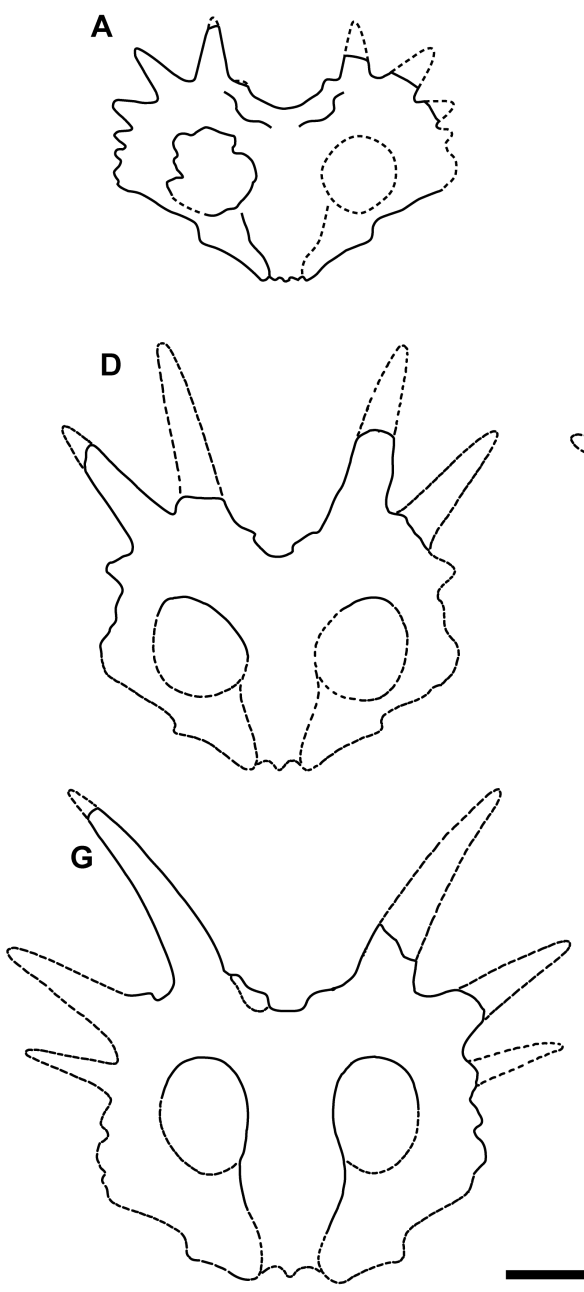

B
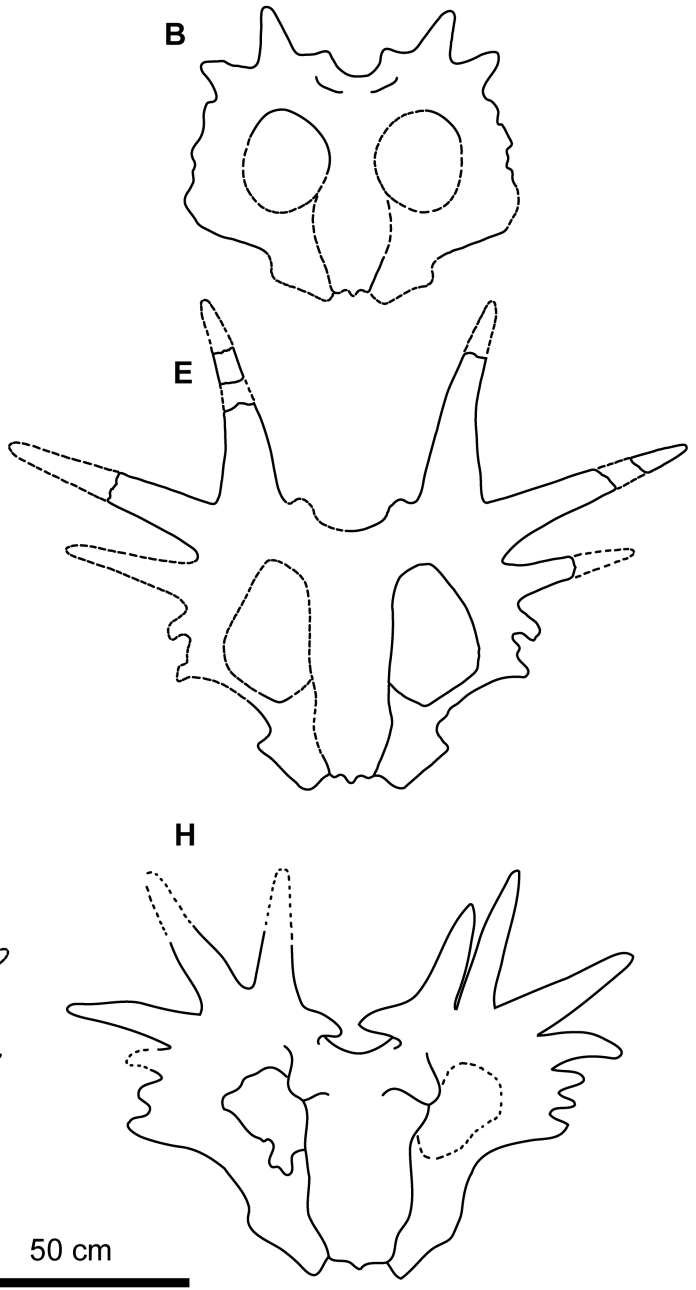
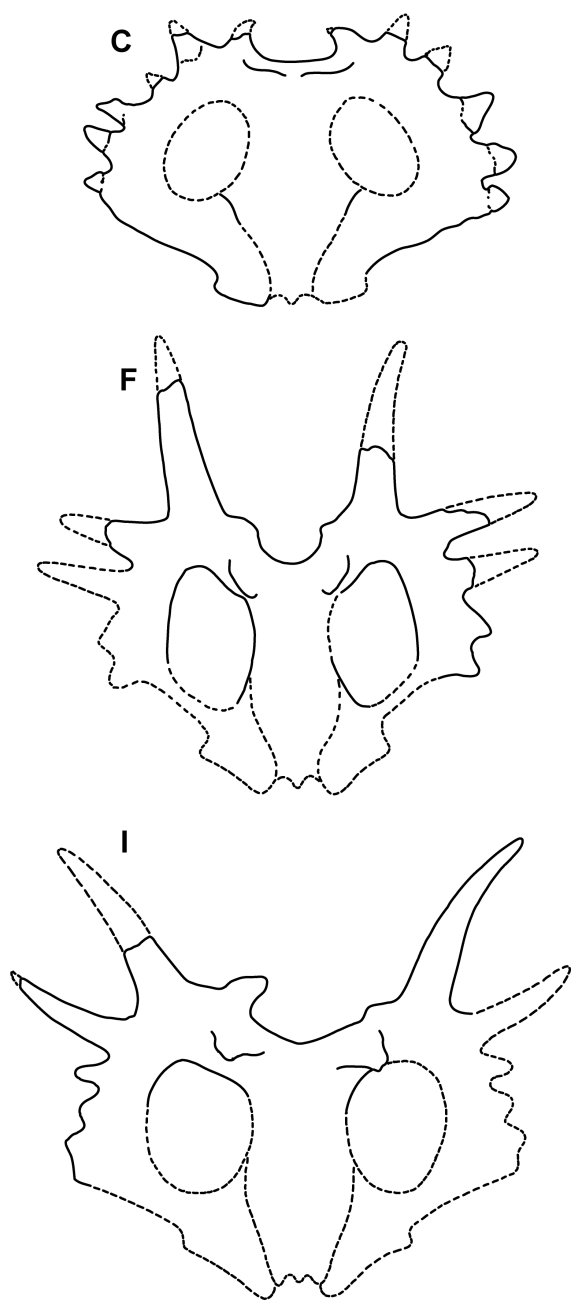

Figure 10. Reconstructed line drawings of the complete or relatively complete parietals of the centrosaurine ceratopsid Styracosaurus albertensis in dorsal (i.e., orthogonal to frill). Specimens are arranged in approximate order of increasing size of parietal ornamentation and size. A, TMP 2009.080.0001. B, TMP 1989.098.0001. C, TMP 1989.126.0001. D, TMP 1988.036.0020. E, CMN 344. F, TMP 2005.012.0058. G, TMP 1987.052.0001. H, UALVP 55900. I, ROM 1436. Based on development of the parietal ornamentation A-C represent subadult specimens, whereas D-I are regarded as largely skeletally mature. Scale bar $=50 \mathrm{~cm}$.

specimens, suggesting an opposite trajectory or variability. Previous work has suggested that the frills of centrosaurines (Sampson et al. 1997), and specifically Centrosaurus apertus (Frederickson and Tumarkin-Deratzian 2014), become wider with age, with narrower morphologies restricted to smaller/younger forms. Sampson et al. (1997) argue that frill width in Centrosaurus is positively allometric, but the sample of very small Centrosaurus frills is limited. Although the smallest frill is distinctly narrow, most of the juvenile ontogenetic series is unknown, and all other specimens in their analysis traditionally identified as Centrosaurus, are adults or old adults (Sampson et al. 1997). None of these larger skulls show such a trend despite differing considerably in size. In the size series of Styracosaurus presented here, however, the smallest/youngest parietals are already broad, and retain broad aspect ratios in the largest specimens, suggesting frill shape may be isometric. Two potential, but not mutually exclusive, conclusions can be drawn from these data. Firstly, it is possible that the frill of Styracosaurus is distinct from Centrosaurus in the allometry of the frill, specifically regarding the length/width ratio. If differences in frill allometry between these taxa exist, they may be reciprocal to the vast differences seen in parietal process allometry. Secondly, it is possible that much of this shape change is restricted to the earliest ontogenetic stages, and frills of $-80 \%$ maximum size or larger (the current Styracosaurus sample) are largely isometric with regard to frill shape. Additional young specimens are required to confirm frill allometry for both genera.

Taken together, the results suggest that there are subtle differences in the ontogeny of Centrosaurus and Styracosaurus, particularly with respect to nasal and postorbital horncores, and 
parietals. It is worth noting that these differences in patterns of ontogenetic development (i.e., heterochrony) between these closely related taxa are largely restricted to the most diagnostic features, which have been hypothesized to be evolving under sociosexual selection (Sampson 1997, 1999; Padian and Horner 2011; Knell et al. 2013; Knapp et al. 2018).

\section{Qualifying Variation in Styracosaurus Parietal}

Processes: Previous work on Styracosaurus albertensis has described significant variation within parietal ornamentation (Ryan et al. 2007; Holmes et al. 2020). This variation in parietal morphology is illustrated in Figures 2 and 10. While some of this variation, specifically the absolute sizes of the parietal processes (e.g., Fig. 10A-C), can be attributed to size and age of the individual (see previous section) not all of it can be explained in this way. Parietal processes 1 and 2 are quite variable in the extent of their expression, from indistinct or non-existent (e.g., CMN 344, TMP 1988.036.0020, 2016.012.0029) to moderate (TMP 1984.093.001, 2005.012.0058), to extensive, approaching Centrosaurus in size and shape (e.g., ROM 1436, UALVP 55900). Despite this variability, when present these processes are consistent in their positions and orientations. Process P1 originates from the anterodorsal surface of the posterior bar of the parietal posterior to the medial margin of the fenestra, and curves anterolaterally from this surface towards the fenestra. The only exception is an anomalous frill from BB 42 (TMP 1981.019.0249), which shows a very large $\mathrm{P} 1$ process in the normal position but projecting posterodorsally and curved posteriorly. Process P2 consistently originates on the posterior aspect of the posterior bar, just posterior to the base of $\mathrm{P} 1$, and projects posteromedially with a medial curvature. Processes P1 and P2 also appear to co-vary in their expression, such that when one is large, so is the other. Process P3 is (with the exception of UALVP 55900) consistently the largest parietal spike, in terms of both length and basal dimensions, and projects largely posteriorly, with a variable amount of curvature, generally laterally. Process P4 is similar to P3 but is generally slightly smaller (in length and basal diameter), projects posterolaterally, and appears to be more variable in both size and orientation than P3. Process P5 is variable in expression and can be a short tab (e.g., ROM 1436) or a true elongate spike (e.g., CMN 344, TMP 2005.012.0058, UALVP 55900). Processes 6, 7, and 8 (if present), are much smaller, and vary from modest scallops (e.g., ROM 1436, TMP 1987.052.001) to moderately sized tabs (e.g., CMN 344, TMP 2005.0012.0058, UALVP 55900).

Given the degree of variation with the parietal processes of Styracosaurus, it is worth considering the potential sources of this variation. Styracosaurus has been demonstrated to replace Centrosaurus within a constrained and well sampled formation. Centrosaurus is diagnosed based in part on large P1 and P2 processes. Because these processes are highly variable in both size and shape in Styracosaurus, it is tempting to hypothesize an anagenic evolutionary trend from Centrosaurus to Styracosaurus, with a decrease in P1 and P2 expression through time. However, when the Styracosaurus specimens are placed in their stratigraphic positions within the formation (Fig. 2), no obvious correlation between $\mathrm{P} 1$ and $\mathrm{P} 2$ expression and stratigraphy is seen. Specimens with well-developed P1 and P2 processes can be found throughout the stratigraphic range of the species, both low (e.g., TMP 2005.012.058) and high (e.g., UAVLP 55900; Holmes et al. 2020). Similarly, specimens with indistinct or non-existent $\mathrm{P} 1$ and $\mathrm{P} 2$ processes can be found throughout the stratigraphic range of the species, both low (e.g., TMP 1987.052.0001) and high (e.g., TMP 1988.036.0020). Perhaps even more telling, extensive samples of parietals from Styracosaurus bonebeds (particularly BB42) show a high degree of variation within a single assemblage representing a herd, or at the very least a penecontemporaneous sample within the species. Frills from BB42 show a spectrum of P1 and P2 development from extensive (e.g., TMP1981.019.0249), to moderate (e.g., TMP 1966.010.0004, 1984.93.0001, 1999.055.0002, $1999.055 .0005,2001.012 .0004)$ to indistinct processes (e.g., TMP 1981.019.0157). These results agree with Holmes et al. (2020) and are inconsistent with the idea that the variability in the frills of Styracosaurus, particularly the relative expression of $\mathrm{P} 1$ and $\mathrm{P} 2$, is due to a directional within-lineage (i.e., anagenesis) evolutionary trend from a Centrosaurus, or Centrosaurus-like, ancestor. This pattern is most consistent with a high level of variation in the parietal processes, particularly the development of P1 and P2, being maintained within the Styracosaurus population being sampled through fossils in the upper Dinosaur Park Formation. Given the current sample, the simplest explanation may be that the frill of Styracosaurus is just more variable in parietal morphology than closely related taxa, specifically Centrosaurus. This higher degree of variation may be due to stronger positive allometry acting on a greater number of parietal processes during growth.

It has been noted previously (Holmes et al. 2020) that parietal spike orientation appears to be correlated with the specific location that each spike occupies on the curved parietal margin, although this relationship was not quantified. In order to test this qualitative impression, we measured and plotted the position and orientation of each parietal spike-see Materials and Methods. The resulting plot (Fig. 11) confirms a close correlation between the position of the base of each epiparietal on the parietal margin and the orientation of its long axis with respect to the midline. Process 1 is the major exception to this pattern, but this may simply be because, unlike the other epiossifications, it 


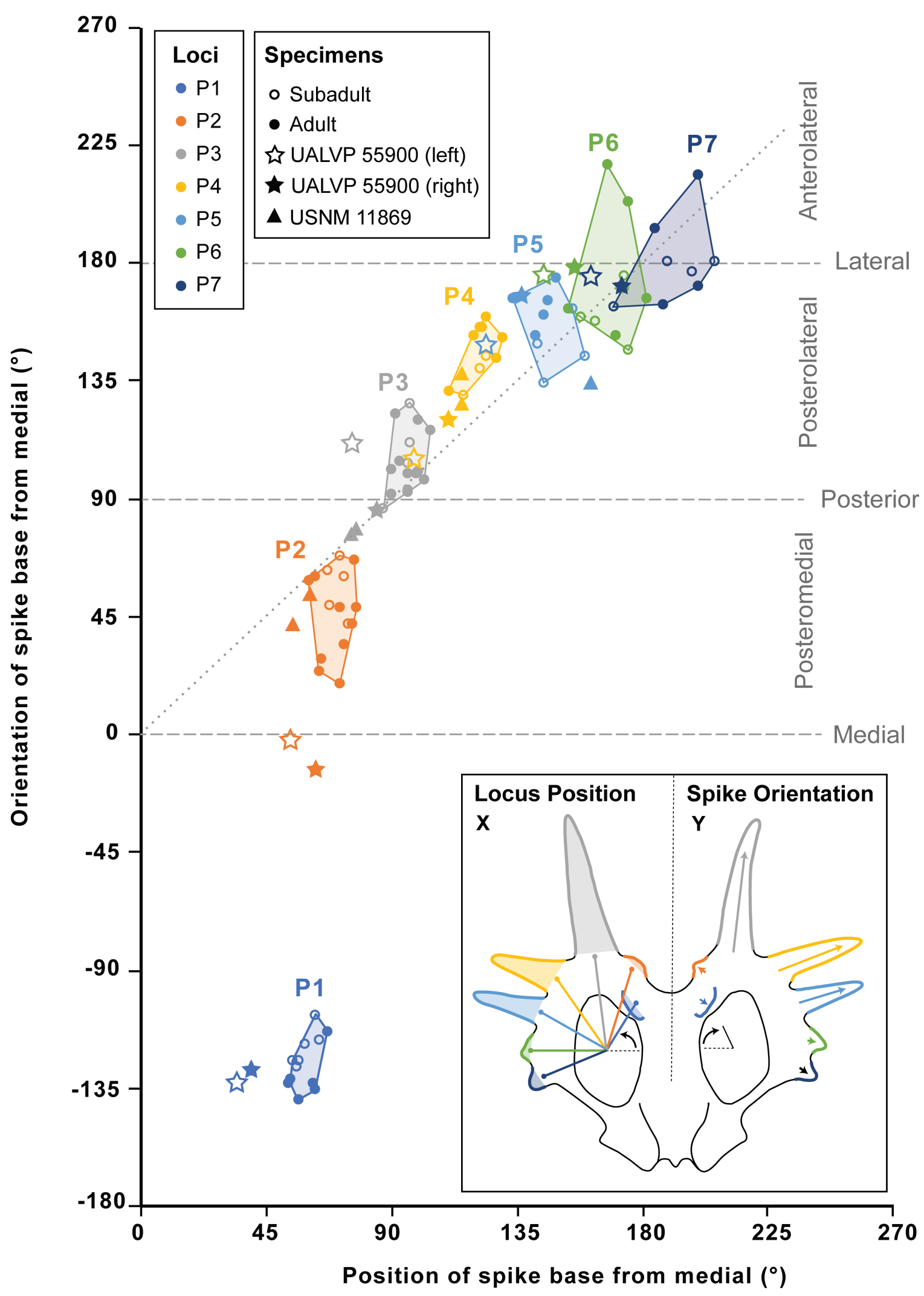

Figure 11. Graph depicting the orientation of each parietal process as a function of its radial position along the margin of the parietal. Serial homologous loci (i.e. P1-P7) are differentiated by colour and clusters are highlighted with convex hulls (excluding UALVP 55900, and USNM 11869). Horizontal dashed lines represent medial, posterior and lateral orientations. Diagonal dotted line represents 1:1 correlation in base opposition and process orientation. Immature specimens indicated with open circles, adult specimens indicated with solid circles, UALVP 55900 indicated with stars and USNM 11869 indicated by triangles. 
originates from the dorsal surface of the parietal rather than from its periphery. The correlation with the remaining parietal loci is nearly 1:1 (a 1:1 relationship is illustrated by the diagonal dotted line in Fig. 11). Even further, homologous loci (e.g., P1, P2) generally plot consistently between specimens (UALVP 5590 is a conspicuous exception - see next paragraph) regardless of the perceived age of the specimen. In other words, the coloured convex hulls generally do not overlap and occupy unique areas. Of interest, parietal processes for subadult frills (i.e., TMP 1988.036.0020, TMP 1989.098.0001, TMP 1989.126.0001) do not show patterns distinct from those of the adult skulls, suggesting both the position of the epiossification loci and the orientation of the spine are conserved through ontogeny (at least through subadult and adult stages) and are therefore reliable taxonomic characters (Fig. 11).

The exception to this correlation between position and orientation is UALVP 55900, especially the left side, where a high degree of asymmetry with respect to the right side has accentuated the effect (Holmes et al. 2020). For P1 and $\mathrm{P} 2$, both left and right loci fall outside the area occupied by the other specimens. For P3, the left locus is an outlier and the right is slightly outside the hull. For P4, the left occurs centrally in the area occupied by $\mathrm{P} 3$ of other specimens, while the right falls between $\mathrm{P} 3$ and P4. A similar situation is seen for $\mathrm{P} 5$, where the left falls in the middle of the $\mathrm{P} 4$ data from the other specimens, and the right falls on the edge of the space occupied by the P5 of other specimens. This pattern continues for P6 and P7. Although the position of the left P3 does not overlap with the space occupied by any of the epiossifications of the other specimens, left P4 is similar to P3, left P5 is similar to P4, left $\mathrm{P} 6$ is similar to $\mathrm{P} 5$ and left $\mathrm{P} 7$ is similar to $\mathrm{P} 6$, while their right counterparts are relatively normal. It is possible that crowding produced by an extra epiossification on the left side (see Holmes et al. 2020) has caused the homologized loci to shift posteromedially along the curve of the parietal margin. Despite this odd asymmetry, the specimen nevertheless is diagnostic to Styracosaurus albertensis, and helps to emphasize the amount of variability and asymmetry that is possible within a clearly diagnosable taxon.

The enigmatic taxon Styracosaurus (=Rubeosaurus) ovatus (USNM 11869) from the upper Two Medicine Formation of Montana was also included in the plot. The positions and orientations of the P2 and $\mathrm{P} 4$ of this specimen do not deviate markedly from the other skulls, but the bases of both P3 loci are positioned farther medial, and their long axes are angled more strongly medially than any of the other skulls. It should be noted that there are now several other Styracosaurus albertensis specimens in which the P3 ossifications are less than $90^{\circ}$ (i.e., oriented slightly medial), including TMP 2009.080.0001 and UAVLVP 55900.
However, USNM 11869 is the most extreme example of this medial projection. If the diagnosis of $S$. ovatus is based only on medially projecting $\mathrm{P} 3$ processes, then this also includes several specimens (i.e., TMP 2009.080.0001 and UAVLVP 55900) that are both morphologically consistent with $S$. albertensis, and derived from the restricted temporal and geographic range of $S$. albertensis. This supports the argument (Holmes et al. 2020) that Styracosaurus ovatus simply represents an extreme morph of $S$. albertensis, and is not a distinct taxon. At the very least, the diagnosis of $S$. ovatus is problematic and cannot rely solely on $\mathrm{P} 3$ processes that project posteromedially.

Discussion of Epiossification Fusion: In other small ceratopsid skulls (e.g., TMP 1989.126.0001, UALVP 40), the epiossifications are highly conspicuous, being little triangular structures attached, but not fused to, the apices of convexities distributed around the margin of the frill. As the skulls grew, the ossifications appear to have grown medially (both dorsally and ventrally) around the convexity, and eventually enveloped it so that the suture between the ossification and squamosal/parietal is located in line with the deepest point in the intervening scallops. Once they were fully coossified, the sutures became obscured. In the skulls where epiossifications are fully fused to the bone surface with the sutures not visible, or where the epiossification is absent, there is little option other than to measure the total distance from the deepest point of the scallop between epiossifications to the tip of the ossification/scallop. This may overestimate the total dimension of the ossification, because in some cases, the base of the ornament is actually formed by the underlying bone (i.e., squamosal or parietal). This appears to be the case in epiparietals 4, 5, and especially P6 of TMP 2009.080.0001. However, in some cases, textural differences between the bone surfaces of epiossifications and underlying bones might allow us to estimate the point of articulation. In TMP 2009.080.0001, P5 has a faint suture that coincides with the transition in texture (epiossification is rough, underlying bone smoother). In this case, the epiossification starts half way up the undulation. To a certain extent this distinction may be moot, as attempts to quantify the size and shape of the epiossification in isolation (i.e., excluding any contribution of the parietal/squamosal) and those investigating the entire process are asking very similar questions in the context of the evolution of these structures.

Although the edges of the squamosals of TMP 2009.080.0001 show the typical 'scalloped' pattern that indicates epiossification loci, there is no evidence of the ossifications themselves. Based on the size of the skull, one would have expected that they would be present, and at least partially fused. It is possible they had not fused to the squamosal at the time of death, and were disassociated 
from the specimen prior to burial. In direct contrast, the epiparietals are not only present, but they are well coossified, to the degree that the sutures are hard to see. The only exception is one epiparietal (between the right P6 and P7) that shows a distinct suture. It is unclear why the states of development of the squamosal and parietal are so out of sync, but for this specimen it seems to be the case. How pervasive this heterochronic pattern is across Styracosaurus or Ceratopsidae, and its potential use in comparing developmental timing is unclear.

Sampson et al. (1997) and Frederickson and TumarkinDeratzian (2014) suggested that both epiparietals and episquamosals of Centrosaurus fuse to the frill in a posterior to anterior sequence. Whatever the case for the sequence of epiparietal fusion in centrosaurines, some Centrosaurus specimens (e.g., UALVP 11735) show the opposite pattern, with the fusion of episquamosals being most advanced anteriorly as in the case of Chasmosaurus (Godfrey and Holmes 1995). Unfortunately, no episquamosals are preserved in TMP 2009.080.0001, so it does not inform this question. In any case, this remains a potentially important question, as Frederickson and Tumarkin-Deratzian (2014) point out, epiossification fusion is a reliable ontogenetic indicator in Centrosaurus, and so potentially it may also be in the closely related Styracosaurus.

\section{CONCLUSIONS}

The Styracosaurus specimen TMP 2009.080.0001, although about $80 \%$ of the size of the largest skull, exhibits many distinctly immature features, in particular small, triangular parietal epiossifications; a small, thin, recurved nasal horncore; and low, rounded postorbital horncores. This indicates that the pronounced cranial ornaments of adult individuals did not develop at a constant rate as the animal grew, but appeared rapidly only during the last stages of ontogeny. Nevertheless, subtle differences between these ossifications (e.g., P3 is measurably larger than any other epiparietal ossification, even in small individuals) should allow immature individuals of Styracosaurus to be identified.

Skull ontogeny of Styracosaurus resembles that of Centrosaurus and centrosaurines in general, but the timing of some events appears to be different, with some being accelerated (e.g., rate of growth as well as fusion of the nasal horncores, timing of cessation of growth of postorbital horncores), others delayed (e.g., development of parietal ornamentation, change in shape of nasal horncore, development of orbital horncores), or truncated (e.g., shape change in nasal horncores). In many cases, ontogenetic changes within one area of the skull (e.g., nasal) have become decoupled from general skull ontogeny, with each feature following its own ontogenetic rate and trajectory. In one case, an intermediate growth stage is eliminated (e.g., loss of the tall, pointed, 'pyramidal' orbital horncores of subadult Centrosaurus), or in another case, reversed (e.g., sagittal bumps on the medial parietal bar of Styracosaurus reduced in size during growth, but became larger in Centrosaurus). Taken together, these suggest that many of the diagnostic differences between Styracosaurus and Centrosaurus are the result of heterochrony of the cranial ornaments.

Parietal ornamentation in Styracosaurus is more variable than in Centrosaurus. In some skulls, P1 can be expressed as a subtle bump on the posteromedial rim of the parietal, while in other skulls, it is prominent, in some cases forming a large anteriorly curving hook. Parietal process 2 is totally absent in a few skulls, but in others, it is expressed as a large, medially curving hook approaching the size and morphology exhibited by Centrosaurus. Process 3 is more consistent in morphology. Although small in small skulls, it exhibits strong positive allometry. It is nearly always the largest epiparietal. Process 4 is slightly smaller than P3, but is generally otherwise similar, although its morphology is more variable. Processes 5-7 also exhibit more variability in both size and morphology. Although sizes of epiparietals appear to correlate positively with skull size, there is no evidence of an evolutionary trend in size or morphology of parietal ornamentation in Styracosaurus.

Parietal epiossification orientation with respect to the midline is quite variable in Styracosaurus, and can be asymmetric in individual skulls. The specific orientations of these ossifications are directly correlated with the position they occupy on the curved margin of the parietal, and neither parameter should be taken in isolation. This relationship is also consistent through ontogeny.

\section{ACKNOWLEDGEMENTS}

Specimen TMP 2009.080.0001 was found by James Wood in 2018 and collected by Darren Tanke, Mark Mitchell, Donald Henderson, and Tai Kubo in 2009. The 2014 and 2016 field crews excavated additional material from Styracosaurus bonebeds BB042, BB130, and BB301, and the 2018 field crew found and excavated TMP 2018.012.0023. The Eastern Irrigation District (EID) provided access to lands for TMP 2005.012.0058, 2009.080.0001, 2018.012.0023, and BB130, and Lee Fryberger and family provided access to lands for the Fryberger Bonebed (BB301). Dinosaur Provincial Park staff provided field logistical support.

TMP 2009.080.0001 was largely prepared by Ian Macdonald and Darren Tanke, with assistance from Dawna Macleod, Judy Graham, and Quintin Pretorius. Collections access and assistance was provided by Brandon Strilisky, 
Rebecca Sanchez, Tom Courtenay, Heather Feeney, and Rhian Russell (RTMP), Howard Gibbins and Clive Coy (UALVP), Margaret Currie and Kieran Shepherd (CMN) and Kevin Seymour and David Evans (ROM), Christina Barron-Ortiz (RAM). Howard Gibbins also provided additional details on UALVP 55900. David Eberth provided field assistance and data in placing specimens into their stratigraphic context. Ben Borkovic, David Eberth, David Evans, Michael Ryan, and Darren Tanke, provided useful discussions on Styracosaurus. Andrew Farke and Catherine Forster provided reviews that significantly improved the manuscript, while Jordan Mallon handled the editorial process. Financial support was for this project was provided by the Royal Tyrrell Museum of Palaeontology and the Royal Tyrrell Museum Cooperating Society.

\section{LITERATURE CITED}

Brown, C.M. 2013. Advances in quantitative methods in dinosaur palaeobiology: a case study in horned dinosaur evolution. PhD Dissertation, Department of Ecology and Evolutionary Biology, University of Toronto, Toronto. $443 \mathrm{pp}$.

Brown, C.M., A.P. Russell, and M.J. Ryan. 2009. Pattern and transition of surficial bone texture of the centrosaurine frill and their ontogenetic and taxonomic implications. Journal of Vertebrate Paleontology 29:132-141.

Currie, P.J., R.B. Holmes, M.J. Ryan, and C. Coy. 2016. A juvenile chasmosaurine ceratopsid (Dinosauria, Ornithischia) from the Dinosaur Park Formation, Alberta, Canada. Journal of Vertebrate Paleontology 36: e1048348.

Dodson, P. 1989. Avaceratops lammersi: a new ceratopsid from the Judith River Formation of Montana. Proceedings of the Academy of Natural Sciences of Philadelphia 138:305-317.

Dodson, P., and P.J. Currie. 1988. The smallest ceratopsid skull - Judith River Formation of Alberta. Canadian Journal of Earth Science 24:926-930.

Evans, D.C. 2007. Ontogeny and evolution of lambeosaurine dinosaurs (Ornithischia: Hadrosauridae). PhD Dissertation, Department of Ecology and Evolutionary Biology, University of Toronto, Toronto, Canada. 497 pp.

Evans, D.C. 2010. Cranial anatomy and systematics of Hypacrosaurus altispinus, and a comparative analysis of skull growth in lambeosaurine hadrosaurids (Dinosauria: Ornithischia). Zoological Journal of the Linnean Society 159:398-434.

Farke, A.A. 2010. Evolution, homology, and function of the supracranial sinuses in ceratopsian dinosaurs. Journal of Vertebrate Paleontology 30:1486-1500.

Farke, A.A., D.J. Chok, A. Herrero, B. Scolieri, and S. Werning. 2013. Ontogeny in the tube-crested dinosaur Parasaurolophus (Hadrosauridae) and heterochrony in hadrosaurids. PeerJ 1: e182.

Frederickson, J.A., and A.R. Tumarkin-Deratzian. 2014.

Craniofacial ontogeny in Centrosaurus apertus. PeerJ 2: e252.
Gilmore, C.W. 1917. Brachyceratops, a ceratopsian dinosaur from the Two Medicine Formation of Montana, with notes on associated fossil reptiles. United States Geological Survey Professional paper 103:1-45.

Gilmore, C.W. 1922. The smallest known horned dinosaur, Brachyceratops. United States National Museum Proceedings 61:1-45.

Gilmore, C.W. 1930. On dinosaurian reptiles from the Two Medicine Formation of Montana. United States National Museum, Proceedings 77:1-39.

Goodwin, M.B., W.A. Clemens, J.R. Horner, and K. Padian. 2006. The smallest known Triceratops skull: New observations on ceratopsid cranial anatomy and ontogeny. Journal of Vertebrate Paleontology 26:103-112.

Griffin, C.T., and S.J. Nesbitt. 2016. Anomalously high variation in postnatal development is ancestral for dinosaurs but lost in birds. Proceedings of the National Academy of Sciences 113:14757-14762.

Holmes, R., and M. Ryan. 2013. The postcranial skeleton of Styracosaurus albertensis. Kirtlandia 58:5-37.

Holmes, R.B., W.S.I. Persons, B.S Rupal, A.J. Qureshi, and P.J. Currie. 2020. Morphological variation and asymmetrical development in the skull of Styracosaurus albertensis. Cretaceous Research 107:16 pp.. DOI 10.1016/j.cretres.2019.104308

Knapp, A., R.J. Knell, A.A. Farke, M.A. Loewen, and D.W. Hone. 2018. Patterns of divergence in the morphology of ceratopsian dinosaurs: sympatry is not a driver of ornament evolution. Proceedings of the Royal Society B: Biological Sciences 285:20180312.

Knell, R.J., D. Naish, J.L. Tomkins, and D.W.E. Hone. 2013. Sexual selection in prehistoric animals: detection and implications. Trends in Ecology \& Evolution 28:38-47.

Lambe, L.M. 1913. A new genus and species of Ceratopsia from the Belly River Formation of Alberta. Ottawa Naturalist 27:109-116.

Mallon, J.C., M.J. Ryan, and J.A. Campbell. 2015. Skull ontogeny in Arrhinoceratops brachyops (Ornithischia: Ceratopsidae) and other horned dinosaurs. Zoological Journal of the Linnean Society 175:910-929.

McDonald, A.T. 2011. A subadult specimen of Rubeosaurus ovatus (Dinosauria: Ceratopsidae), with observations on other ceratopsids from the Two Medicine Formation. PLoS ONE 6: e22710.

Padian, K., and J.R. Horner. 2011. The evolution of 'bizarre structures' in dinosaurs: biomechanics, sexual selection, social selection or species recognition? Journal of Zoology 283:3-17.

Penkalski, P., and P. Dodson. 1999. The morphology and systematics of Avaceratops, a primitive horned dinosaur from the Judith River Formation (late Campanian) of Montana, with the description of a second skull. Journal of Vertebrate Paleontology 19:692-711.

Petermann, H., N.M. Koch, and J.A. Gauthier. 2017. Osteohistology and sequence of suture fusion reveal complex environmentally influenced growth in the teiid liz- 
ard Aspidoscelis tigris_-Implications for fossil squamates.

Palaeogeography, Palaeoclimataology, Palaeoecology 475:12-22.

Ryan, M.J. 1992. The taphonomy of a Centrosaurus

(Ornithischia: Ceratopsidae) bone bed (Campanian), Dinosaur

Provincial Park, Alberta, Canada. Masters thesis, Department of Biological Sciences. University of Calgary, Calgary. 526 pp.

Ryan, M.J. 2007. A new basal centrosaurine ceratopsid from the Oldman Formation, Southeastern Alberta. Journal of Paleontology 81:376-396.

Ryan, M.J., D.C. Evans, and K.M. Shepherd. 2012. A new ceratopsid from the Foremost Formation (middle Campanian) of Alberta. Canadian Journal of Earth Sciences 49:1251-1262.

Ryan, M.J., R. Holmes, and A.P. Russell. 2007. A revision of the late Campanian centrosaurine ceratopsid genus Styracosaurus from the Western Interior of North America. Journal of Vertebrate Paleontology 27:944-962.

Sampson, S. 1993. Cranial Ornamentations in Ceratopsid Dinosaurs: Systematic, Behavioural, and Evolutionary Implications. PhD Dissertation, Department of Zoology, University of Toronto, Toronto. 299 pp.

Sampson, S.D. 1995. Two new horned dinosaurs from the Upper Cretaceous Two Medicine Formation of Montana: With a phylogenetic analysis of the Centrosaurinae (Ornithischia: Ceratopsidae). Journal of Vertebrate Paleontology 15:743-760.
Sampson, S.D. 1997. Bizarre structures and dinosaur evolution; pp. 39-45 in Dinofest International Proceedings. Academy of Natural Sciences and Arizona State University.

Sampson, S.D. 1999. Sex and destiny: the role of mating signals in speciation and macroevolution. Historical Biology 13:173-197.

Sampson, S.D., M.J. Ryan, and D.H. Tanke. 1997. Craniofacial ontogeny in centrosaurine dinosaurs (Ornithischia: Ceratopsidae): taxonomic and behavioral implications. Zoological Journal of the Linnean Society 121:293-337.

Scannella, J.B., D.W. Fowler, M.B. Goodwin, and J.R. Horner. 2014. Evolutionary trends in Triceratops from the Hell Creek Formation, Montana. Proceedings of the National Academy of Sciences 111:10245-10250.

Sternberg, C.M. 1927. Horned dinosaur group in the National Museum of Canada. Canadian Field Naturalist 41:67-73.

Tokaryk, T.T. 1997. First evidence of juvenile ceratopsians (Reptilia: Ornithischia) from the Frenchman Formation (late Maastrichtian) of Saskatchewan. Canadian Journal of Earth Sciences 34:1401-1404.

Tumarkin-Deratzian, A.B. 2010. Histological evaluation of ontogenetic bone surface texture changes in the frill of Centrosaurus apertus; pp. 251-263 in M.J. Ryan, B.J. Chinnery-Algeier, and D.A. Eberth (eds.), New Perspectives on Horned Dinosaurs, the Royal Tyrrell Museum Ceratopsian Symposium. Indiana University Press, Bloomington. 


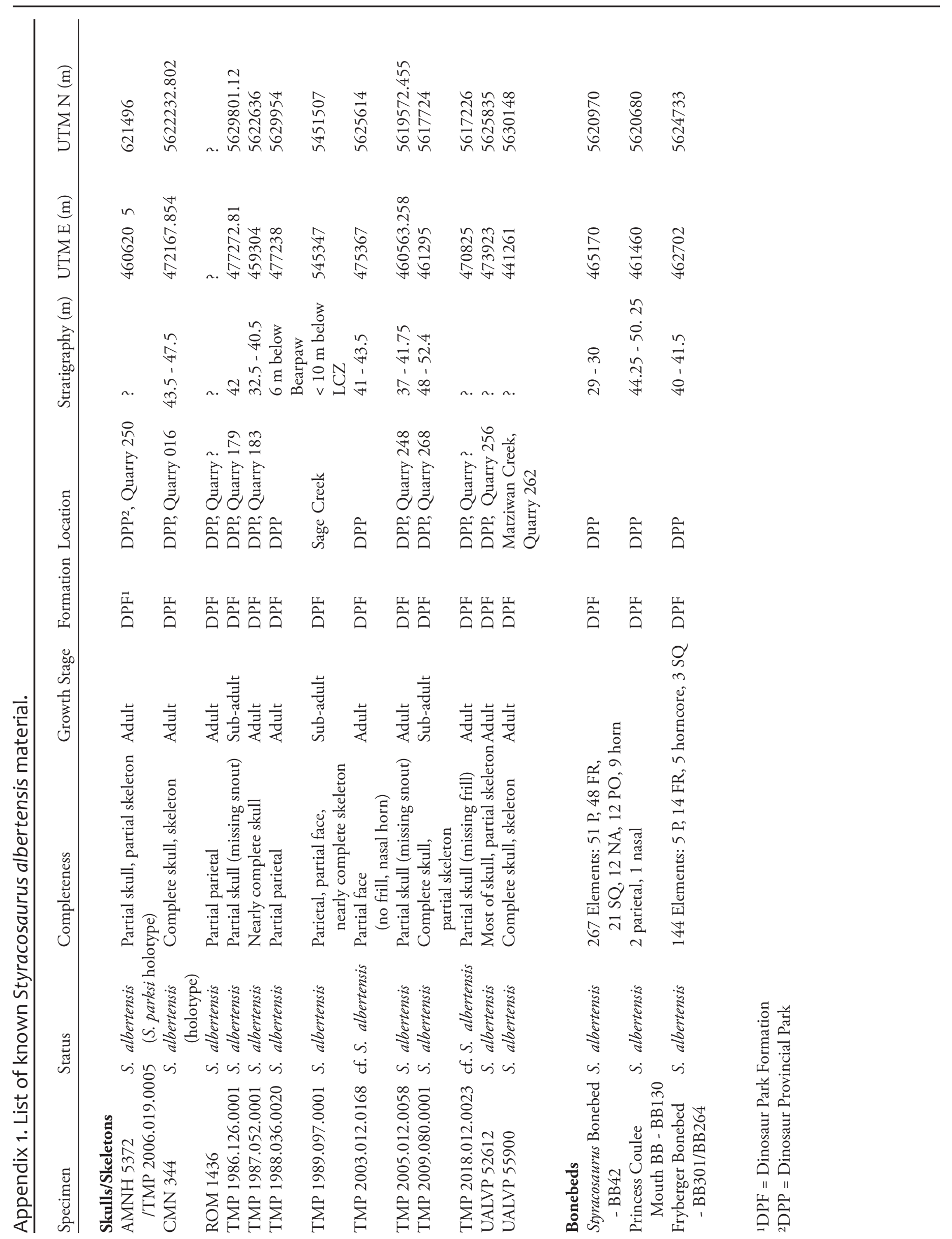


Appendix 2. Radial position of base (position, ${ }^{\circ}$ ) and orientation from base to apex (projection, ${ }^{\circ}$ ) of each parietal spike for reasonably complete parietals of Styracosaurus, as plotted in Figure 11. The circular coordinate takes origin at the centre of the fenestra, with $0^{\circ}$ orientated medial, $90^{\circ}$ oriented posterior, and $180^{\circ}$ oriented lateral. See inset of Figure 11 for diagram.

\begin{tabular}{|c|c|c|c|c|c|c|c|c|c|c|}
\hline $\begin{array}{l}\text { Parietal } \\
\text { Horn ID }\end{array}$ & \multicolumn{2}{|c|}{ TMP 1989.097.0001 } & TMP 1986.126.0001 & $\begin{array}{l}86.126 .0001 \\
\text { Projection }\end{array}$ & $\begin{array}{l}\text { ROM } 1 \\
\text { Postion }\end{array}$ & $\begin{array}{l}436 \\
\text { Projection }\end{array}$ & $\begin{array}{l}\text { CMN } 3 \\
\text { Postion }\end{array}$ & $\begin{array}{l}44 \\
\text { Projection }\end{array}$ & \multicolumn{2}{|c|}{ Postion Projection } \\
\hline & 59 & 241 & 57 & 235 & 67 & 246 & - & - & 62 & 226 \\
\hline P1 L & 64 & 243 & 63 & 252 & 63 & 224 & - & - & 54 & 228 \\
\hline P2 R & 67 & 62 & 72 & 68 & 73 & 34 & - & - & 72 & 48 \\
\hline P2 L & 73 & 60 & 75 & 42 & 76 & 42 & 77 & 66 & 65 & 28 \\
\hline P3 R & 96 & 103 & 97 & 126 & 104 & 116 & 100 & 100 & 96 & 99 \\
\hline P3 L & 97 & 111 & 99 & - & 100 & 120 & 99 & 99 & 90 & 91 \\
\hline P4 R & 124 & 144 & 125 & - & 130 & 151 & 123 & 155 & 124 & 159 \\
\hline P4 L & 122 & 139 & 124 & - & - & - & 122 & 155 & 120 & 152 \\
\hline P5 R & 145 & 134 & 155 & 162 & 149 & 174 & - & - & - & - \\
\hline P5 L & 143 & 149 & 160 & 144 & - & - & 146 & 165 & 145 & 160 \\
\hline P6 R & 174 & 175 & 175 & 146 & 171 & 152 & - & - & - & - \\
\hline P6 L & 163 & 157 & 174 & 170 & - & - & 168 & 217 & 175 & 203 \\
\hline P7 R & 189 & 180 & 198 & 176 & 188 & 164 & - & - & - & - \\
\hline P7 L & NA & $\mathrm{NA}$ & 206 & 180 & - & - & 185 & 193 & 200 & 213 \\
\hline Parietal & \multicolumn{2}{|c|}{ TMP 1987.051.0001 } & \multicolumn{2}{|c|}{ TMP 1988.036.0020 } & \multicolumn{2}{|c|}{ TMP 1999.055.0005 } & \multicolumn{2}{|c|}{ TMP 1984.043.0001 } & \multicolumn{2}{|c|}{ TMP 2009.080.0001 } \\
\hline Horn ID & Postion & Projection & Postion & n Projection & Postion & Projection & Postion & Projection & Postion & Projection \\
\hline P1 R & & & & & 245 & & 57 & 220 & 55 & 235 \\
\hline P1 L & & & & & 241 & & 53 & 226 & 56 & 233 \\
\hline P2 R & 63 & 60 & 64 & 24 & 72 & 19 & 68 & & 68 & 49 \\
\hline P2 L & & & 61 & 58 & 78 & 48 & & & 69 & \\
\hline P3 R & 92 & 122 & 88 & & 96 & 93 & 96 & 92 & 87 & 86 \\
\hline P3 L & 93 & 104 & 90 & 101 & 102 & 97 & & & 87 & \\
\hline P4 R & & & 111 & 131 & & & & & 116 & 129 \\
\hline P4 L & 128 & 143 & 114 & & & & & & 114 & \\
\hline P5 R & & & 134 & 166 & & & & & 142 & 152 \\
\hline P5 L & 157 & & & & & & & & & 141 \\
\hline P6 R & & & 154 & 162 & & & & & 158 & 159 \\
\hline P6 L & 182 & 166 & & & & & & & 157 & \\
\hline P7 R & & & & & & & & & 170 & 163 \\
\hline P7 L & 200 & 171 & & & & & & & & \\
\hline Parietal & USNM & 11869 & UALVP 5 & 55900 & & & & & & \\
\hline Horn ID & Postion & Projection & Postion & Projection & & & & & & \\
\hline P1 R & & & 40 & 231 & & & & & & \\
\hline P1 L & & & 35 & 226 & & & & & & \\
\hline P2 R & 61 & 53 & 63 & 346 & & & & & & \\
\hline P2 L & 55 & 41 & 54 & 357 & & & & & & \\
\hline P3 R & 78 & 77 & 85 & 85 & & & & & & \\
\hline P3 L & 76 & 75 & 76 & 110 & & & & & & \\
\hline P4 R & 115 & 137 & 111 & 120 & & & & & & \\
\hline P4 L & 115 & 125 & 98 & 105 & & & & & & \\
\hline P5 R & & & 137 & 167 & & & & & & \\
\hline P5 L & 162 & 133 & 124 & 148 & & & & & & \\
\hline P6 R & & & 156 & 178 & & & & & & \\
\hline P6 L & & & 145 & 175 & & & & & & \\
\hline P7 R & & & 173 & 171 & & & & & & \\
\hline P7 L & & & 162 & 174 & & & & & & \\
\hline P8 R & & & & & & & & & & \\
\hline P8 L & & & 175 & 188 & & & & & & \\
\hline
\end{tabular}


Appendix 3. Specimen numbers for nasal and postorbital horncores included in Figures 7 and 8.

\begin{tabular}{|c|c|c|c|c|c|c|c|c|c|c|}
\hline \multicolumn{11}{|c|}{ Nasals } \\
\hline$\#$ & Specimen \# & Basal Size & Fusion & Reflected & $\#$ & Specimen \# & Basal Size & Fusion & \multicolumn{2}{|c|}{ Reflected } \\
\hline 1 & TMP1995.400.0074 & 72 & unfused & yes & 54 & ТМР1998.093.0163 & 77 & unfuse & & no \\
\hline 2 & TMP1996.012.0286 & 81 & unfused & no & 55 & ТМР2009.031.0001 & 190 & fused, & isolated & yes \\
\hline 3 & TMP1996.012.0288 & 82 & unfused & no & 56 & TMP2009.080.0001 & 191 & fused, & articuated & d no \\
\hline 4 & TMP2009.400.0004 & 104 & unfused & yes & 57 & TMP1966.010.0023 & 194 & fused, & Isolated & no \\
\hline 5 & TMP1995.400.0265 & 117 & unfused & yes & 58 & TMP1966.010.0019 & 212 & fused, & Isolated & yes \\
\hline 6 & TMP1992.036.0442 & 134 & unfused & no & 59 & ТМР1966.010.0021 & 213 & fused, & Isolated & yes \\
\hline 7 & TMP1981.026.0003 & 139 & unfused & yes & 60 & ТМР2017.023.0016 & 223 & fused, & associated & \\
\hline 8 & TMP2014.017.0064 & 147 & unfused & yes & 61 & TMP2005.012.0058 & 225 & fused, & articuated & d yes \\
\hline 9 & TMP1982.018.0220 & 166 & unfused & no & 62 & TMP1966.010.0020 & 226 & fused, & Isolated & yes \\
\hline 10 & TMP1993.036.0435 & 167 & unfused & yes & 63 & TMP1966.010.0022 & 250 & fused, & Isolated & yes \\
\hline 11 & TMP2009.039.0365 & 168 & unfused & no & 64 & UAVLP 55900 & 257 & fused, & articuated & d no \\
\hline 12 & TMP1995.400.0187 & 140 & tip fused & yes & 65 & TMP2018.012.0023 & 259 & fused, & associated & d no \\
\hline 13 & TMP1995.401.0084 & 143 & tip fused & no & 66 & UALVP 52612 & 264 & fused, & articuated & d yes \\
\hline 14 & TMP2016.016.0002 & 158 & tip fused & yes & 67 & CMN 344 & 283 & fused, & articuated & d no \\
\hline 15 & TMP1966.033.0017 & 165 & tip fused & yes & 68 & TMP1987.052.0001 & 321 & fused, & articuated & d yes \\
\hline 16 & TMP1979.011.0083 & 167 & tip fused & yes & & & & & & \\
\hline 17 & TMP1997.145.0074 & 172 & tip fused & no & \multicolumn{2}{|c|}{ Postorbitals } & \multirow{3}{*}{$\begin{array}{l}\text { Basal Side } \\
\text { Size }\end{array}$} & & & \\
\hline 18 & TMP1980.024.0004 & 173 & tip fused & no & $\#$ & Specimen \# & & Fusion & Res. Pit & Refl'd \\
\hline 19 & TMP2016.016.0029 & 176 & tip fused & no & & & & & & \\
\hline 20 & TMP1995.175.0019 & 179 & tip fused & no & & & & & & \\
\hline 21 & TMP1981.022.0010 & 185 & tip fused & no & 1 & TMP1980.016.1694 & 22 right & unfused & no & no \\
\hline 22 & TMP1980.018.0310 & 192 & tip fused & no & 2 & TMP1979.011.0157 & 38 left & unfused & no & yes \\
\hline 23 & TMP1982.018.0044 & 198 & fused, Isolated & no & 3 & TMP1980.018.0016 & 39 right & unfused & no & no \\
\hline 24 & TMP1987.018.0039 & 207 & fused, Isolated & no & 4 & TMP1982.018.0139 & 47 left & unfused & no & yes \\
\hline 25 & TMP1965.023.0019 & 220 & fused, Isolated & no & 5 & TMP1995.400.0164 & 52 left & unfused & no & yes \\
\hline 26 & TMP1981.018.0183 & 222 & fused, Isolated & no & 6 & TMP1995.400.0114 & 53 left & unfused & no & yes \\
\hline 27 & TMP1982.018.0281 & 222 & fused, Isolated & yes & 7 & TMP1979.011.0117 & 74 right & unfused & no & no \\
\hline 28 & TMP1995.401.0045 & 223 & fused, Isolated & no & 8 & TMP1981.022.0013 & 74 right & unfused & no & no \\
\hline 29 & TMP1987.018.0020 & 224 & fused, Isolated & yes & 9 & TMP1995.400.0256 & 78 right & unfused & no & no \\
\hline 30 & TMP1993.036.0587 & 226 & fused, Isolated & no & 10 & ТМР1992.036.0398 & 78 right & unfused & no & no \\
\hline 31 & TMP1982.018.0067 & 230 & fused, Isolated & yes & 11 & TMP1980.016.1043 & 80 right & unfused & no & no \\
\hline 32 & TMP1991.018.0090 & 246 & fused, Isolated & no & 12 & TMP1995.401.0107 & 80 right & unfused & no & no \\
\hline 33 & TMP1992.036.0712 & 247 & fused, Isolated & yes & 13 & TMP1979.011.0020 & 82 left & unfused & no & yes \\
\hline 34 & RAM P 64.5.191 & 248 & fused, Isolated & no & 14 & TMP1979.011.0100 & 85 left & unfused & no & yes \\
\hline 35 & TMP1994.012.0525 & 255 & fused, Isolated & no & 15 & TMP1989.018.0064 & 88 right & unfused & no & no \\
\hline 36 & TMP 1992.082.0001 & 192 & fused, articuated & yes & 16 & TMP2008.079.0047 & 88 right & unfused & no & no \\
\hline 37 & ROM 767 & 195 & fused, articuated & no & 17 & TMP2013.044.0027 & 89 right & unfused & no & no \\
\hline 38 & CMN 8798 & 201 & fused, articuated & yes & 18 & ТМР1994.012.0942 & 90 right & unfused & no & no \\
\hline 39 & CMN 437 (LAVAL) & 206 & fused, articuated & yes & 19 & TMP1986.018.0058 & 93 left & isolated & no & yes \\
\hline 40 & YPM 2015 & 206 & fused, articuated & yes & 20 & TMP1997.012.0192 & 93 right & unfused & no & no \\
\hline 41 & TMP1980.024.0004 & 211 & fused, articuated & yes & 21 & TMP1992.036.1017 & 98 left & unfused & no & yes \\
\hline 42 & TMP1993.036.0117 & 235 & fused, articuated & no & 22 & TMP1982.018.0017 & 105 left & unfused & no & yes \\
\hline 43 & CMN 8795 & 235 & fused, articuated & yes & 23 & ТМР2015.059.0023 & 105 left & partial & no & yes \\
\hline 44 & TMP1994.182.0001 & 241 & fused, articuated & no & 24 & TMP1998.093.0034 & 109 left & fused & no & yes \\
\hline 45 & TMP2006.025.0001 & 252 & fused, articuated & & 25 & TMP2005.009.0007 & $111 \mathrm{left}$ & unfused & no & yes \\
\hline 46 & TMP2015.018.0014 & 258 & fused, articuated & yes & 26 & TMP1994.012.0154 & 111 left & unfused & no & yes \\
\hline 47 & AMNH 5351 & 259 & fused, articuated & no & 27 & TMP1979.011.0163 & 125 right & fused & no & no \\
\hline 48 & UALVP 11735 & 202 & fused, articuated & no & 28 & TMP1994.012.0524 & 130 right & unfused & no & no \\
\hline 49 & CMN 11837 & 214 & fused, articuated & yes & 29 & TMP1997.012.0213 & 135 left & fused & no & yes \\
\hline 50 & CMN 348 & 232 & fused, articuated & yes & 30 & ROM 767 & 144 both & fused & no & no \\
\hline 51 & ROM 43214 & 242 & fused, articuated & yes & 31 & TMP1982.018.0104 & 145 left & fused & no & yes \\
\hline 52 & AMNH 5239 & 264 & fused, articuated & no & 32 & TMP1988.036.0269 & 150 right & fused & no & no \\
\hline 53 & TMP1997.085.0001 & 267 & fused, articuated & yes & 33 & ТМР2014.017.0054 & 150 left & fused & no & yes \\
\hline
\end{tabular}


Brown et al. - Subadult Styracosaurus albertensis

Appendix 3 continued

\begin{tabular}{|c|c|c|c|c|c|c|c|c|c|c|c|c|c|}
\hline $\begin{array}{l}\text { Po } \\
\#\end{array}$ & $\begin{array}{l}\text { torbitals } \\
\text { Specimen \# }\end{array}$ & $\begin{array}{l}\text { Basal } \\
\text { Size }\end{array}$ & Side & Fusion Res. & Pit & Refl'd & \# & Specimen \# & $\begin{array}{l}\text { Basal } \\
\text { Size }\end{array}$ & Side & Fusion $\mathrm{R}$ & Res. Pit & Refl'd \\
\hline 34 & CMN 8797 & 166 & both & fused & no & yes & 75 & TMP1980.018.0083 & 136 & left & fused & yes & yes \\
\hline 35 & CMN 348 & 169 & both & fused & yes & yes & 76 & ТМР1989.018.0040 & 137 & left & fused & yes & yes \\
\hline 36 & UALVP 11735 & 177 & both & fused & no & no & 77 & ТМР1979.011.0041 & 139 & left & fused & yes & yes \\
\hline 37 & TMP1982.018.0262 & 93 & left & unfused & no & yes & 78 & ТМР2014.017.0011 & 139 & left & fused & yes & yes \\
\hline 38 & TMP1979.011.0066 & 94 & right & unfused & no & no & 79 & TMP1979.011.0085 & 141 & left & fused & yes & yes \\
\hline 39 & ТМР2014.017.0024 & 101 & right & uncertain & no & no & 80 & TMP1980.016.0515 & 147 & left & fused & yes & yes \\
\hline 40 & TMP1980.018.0315 & 107 & left & unfused & no & yes & 81 & TMP1979.011.0040 & 155 & right & fused & yes & no \\
\hline 41 & TMP2014.017.0008 & 115 & right & fused & no & no & 82 & TMP1995.012.0145 & 157 & right & fused & yes & no \\
\hline 42 & TMP1979.011.0031 & 116 & left & unfused & no & yes & 83 & TMP1980.018.0303 & 158 & right & fused & yes & no \\
\hline 43 & TMP1980.018.0295 & 117 & left & fused & no & yes & 84 & YPM 2015 & 160 & both & fused & yes & yes \\
\hline 44 & TMP2014.017.0030 & 121 & left & partial & no & yes & 85 & TMP1994.182.0001 & 164 & both & fused & yes & no \\
\hline 45 & TMP1994.012.0940 & 127 & left & partial & no & yes & 86 & TMP1993.070.0001 & 167 & both & fused & yes & yes \\
\hline 46 & TMP1979.011.0120 & 137 & right & fused & no & no & 87 & CMN 8795 & 173 & both & fused & yes & yes \\
\hline 47 & TMP1982.018.0002 & 138 & left & fused & no & yes & 88 & TMP1982.019.0244 & 106 & right & fused & yes & no \\
\hline 48 & TMP2016.016.0031 & 144 & right & fused & no & no & 89 & TMP1979.010.0005 & 108 & both & fused & yes & no \\
\hline 49 & TMP1995.401.0044 & 154 & left & fused & no & yes & 90 & TMP1979.011.0080 & 116 & right & fused & yes & no \\
\hline 50 & TMP1994.012.0158 & 93 & left & fused & yes & yes & 91 & TMP 1965.012.0005 & 130 & right & fused & yes & no \\
\hline 51 & TMP1980.016.1677 & 101 & right & unfused & no & no & 92 & NHM R 4859 & 131 & both & fused & yes & yes \\
\hline 52 & TMP1980.018.0309 & 105 & left & fused & no & yes & 93 & TMP1965.023.0027 & 134 & right & fused & yes & no \\
\hline 53 & TMP1988.036.0033 & 107 & right & unfused & no & no & 94 & TMP2015.024.0069 & 137 & left & fused & yes & yes \\
\hline 54 & TMP1989.018.0009 & 109 & left & fused & no & yes & 95 & AMNH 5351 & 151 & both & fused & yes & no \\
\hline 55 & TMP1980.018.0221 & 119 & right & partial & no & no & 96 & USNM 12742 & 160 & left & fused & yes & yes \\
\hline 56 & TMP1979.011.0084 & 122 & left & fused & no & yes & 97 & CMN 347 & 169 & both & fused & yes & no \\
\hline 57 & CMN 8798 & 124 & both & fused & no & yes & 98 & TMP1997.085.0001 & 185 & both & fused & yes & yes \\
\hline 58 & TMP1980.018.0350 & 127 & left & fused & no & yes & 99 & TMP2014.015.0084 & 63 & left & unfused & no & yes \\
\hline 59 & TMP1992.036.0650 & 128 & left & fused & no & yes & 100 & TMP2009.031.0012 & 95 & right & unfused & d no & no \\
\hline 60 & TMP1979.011.0128 & 135 & left & fused & no & yes & 101 & TMP1998.093.0064 & 95 & right & partial & no & no \\
\hline 61 & TMP1979.011.0129 & 136 & left & fused & no & yes & 102 & TMP2009.080.0001 & 109 & both & partial & no & no \\
\hline 62 & TMP1994.012.0838 & 138 & right & fused & no & no & 103 & TMP2018.012.0013 & 109 & left & fused & no & yes \\
\hline 63 & TMP1982.016.0178 & 145 & right & fused & no & no & 104 & TMP1986.126.0001 & 109 & both & fused & no & yes \\
\hline 64 & TMP1979.011.0081 & 145 & right & fused & no & no & 105 & TMP1966.010.0041 & 109 & left & fused & no & yes \\
\hline 65 & TMP1995.401.0004 & 146 & right & fused & certain & no & 106 & TMP2014.015.0143 & 120 & left & partial & no & yes \\
\hline 66 & TMP1989.018.0024 & 149 & left & fused & no & yes & 107 & TMP2007.012.0059 & 123 & left & fused & no & yes \\
\hline 67 & TMP1979.011.0089 & 154 & right & fused & no & no & 108 & TMP2003.012.0168 & 124 & left & fused & no & yes \\
\hline 68 & AMNH 5429 & 162 & both & fused & no & no & 109 & TMP1990.058.0004 & 120 & right & fused & yes & no \\
\hline 69 & AMNH 5239 & 167 & both & fused & no & yes & 110 & CMN344 & 123 & both & fused & yes & yes \\
\hline 70 & TMP1986.018.0050 & 116 & left & fused & yes & yes & 111 & TMP2002.070.0001 & 124 & left & fused & yes & yes \\
\hline 71 & TMP1967.020.0234 & 117 & right & fused & yes & no & 112 & UALVP 52612 & 125 & both & fused & yes & yes \\
\hline 72 & TMP1992.082.0001 & 119 & left & fused & yes & yes & 113 & TMP2005.012.0058 & 126 & right & fused & yes & no \\
\hline 73 & TMP1986.018.0101 & 120 & left & fused & yes & yes & 114 & UALVP 55900 & 140 & both & fused & yes & no \\
\hline 74 & TMP1983.018.0037 & 135 & left & fused & yes & yes & 115 & TMP2014.015.0094 & 149 & right & fused & yes & no \\
\hline
\end{tabular}

